\title{
Robust energy-based model updating framework for random processes in dynamics: application to shaking-table experiments
}

\author{
M. Diaz ${ }^{\mathrm{a}, \mathrm{b}, *}$, P.-É. Charbonnel ${ }^{\mathrm{b}}$, L. Chamoin ${ }^{\mathrm{a}, \mathrm{c}}$ \\ ${ }^{a}$ Université Paris-Saclay, ENS Paris-Saclay, CNRS, LMT - Laboratoire de Mécanique et Technologie, \\ 91190, Gif-sur-Yvette, France \\ ${ }^{b}$ DES-Service d'Études Mécaniques et Thermiques (SEMT), CEA, Université Paris-Saclay, 91191 \\ Gif-sur-Yvette, France \\ ${ }^{c}$ Institut Universitaire de France (IUF), 1 rue Descartes, 75231 Paris CEDEX 5, France
}

\begin{abstract}
This paper presents a robust model updating strategy for correcting finite element models from datasets acquired in low-frequency dynamics. The proposed methodology is based on the minimization of a modified Constitutive Relation Error (mCRE) made of two terms: (i) a Hermitian data-to-model distance written in the frequency domain enriched with (ii) a CRE residual accounting for model bias with strong mechanical content. An automated L-curve based methodology is derived for tuning the relative weight of the two terms and improving the algorithm robustness to noise level. An extended formulation of the mCRE in terms of Power Spectral Density is also proposed: a data windowing preprocessing step ensures statistical consistency of the updated parameters when dealing with noisy random processes. The methodology is applied to two earthquake engineering examples. The performances of the methodology are assessed using synthetic measurements from a plane frame subjected to random ground acceleration. Actual measurements from the SMART2013 database are next processed to observe the eigenfrequency drop of a reinforced-concrete structure submitted to a sequence of gradually damaging shaking-table tests. In this last application, the corrected model predictions are in good correlation with former data-driven subspace-based identification results.
\end{abstract}

Keywords:

Model Updating, Low-Frequency Dynamics, Modified Constitutive Relation Error (mCRE), Low signal-to-noise ratio measurements, Earthquake Engineering, SMART2013 shaking-table tests

${ }^{*}$ Corresponding author

Email address: matthieu.diaz@ens-paris-saclay.fr (M. Diaz) 


\section{Nomenclature}

\section{Model updating framework}

$\mathcal{M} \quad$ Model operator

(e) Set of model inputs

(y) Set of measurements to be compared to model predictions

$\Pi \circ \quad$ Extraction operator for comparison between model and measurements

$p \quad$ Set of parameters to update of dimension $N$

$\hat{p} \quad$ Optimal parameter estimate obtained after model updating

$\mathcal{P} \quad$ Parameter space, subset of $\mathbb{R}^{N}$

$\mathcal{J} \quad$ Deterministic cost-function to minimize

$\|\bullet\|$ Data-to-model distance norm (non-necessary Euclidean)

$\mathcal{G}$ Tikhonov regularization term

$\alpha \quad$ Weighting coefficient associated to the Tikhonov regularization

$\delta \quad$ Signal-to-noise ratio

\section{Reference dynamical problem}

$\Omega \quad$ Physical domain

$\partial \Omega \quad$ Boundary of the physical domain

$\partial_{1} \Omega \quad$ Boundary of the domain where displacement is imposed

$u_{d} \quad$ Displacement field imposed on $\partial_{1} \Omega$

$\partial_{2} \Omega \quad$ Boundary of the domain where surface loads are applied

$F_{d} \quad$ Surface load applied on $\partial_{2} \Omega$

$n \quad$ Outer normal vector

$f_{d} \quad$ Body forces applied in $\Omega$

$[0 ; T] \quad$ Time domain

$u_{0} \quad$ Initial displacement field

$\dot{u}_{0} \quad$ Initial velocity field

$u \quad$ Displacement field

$\mathcal{U}^{[0 ; T]} \quad$ Space of kinematically admissible displacement fields

$\sigma \quad$ Stress field

$\sigma^{e}, \sigma^{d} \quad$ Elastic and dissipative parts of the stress field

$\mathcal{S}^{[0 ; T]} \quad$ Space of dynamically admissible stress fields

$\varepsilon \quad$ Linearized strain tensor

$\mathcal{K} \quad$ Hooke's elasticity fourth-order tensor

$\mathcal{D} \quad$ Dissipation fourth-order tensor

$\rho \quad$ Volumic mass 


\section{Finite element discretized problem}

$U \quad$ Discrete nodal field associated to $u$

$\bigoplus \quad$ FE assembly operator

$K, D, M \quad$ Stiffness, damping and mass FE matrices

$F \quad$ Discretized force vector

\section{From time domain to frequency domain}

$\begin{array}{cl}t & \text { Time } \\ \omega & \text { Frequency pulsation } \\ Y(\omega) & \text { Continuous frequency counterpart of } y(t) \text { at pulsation } \omega \\ Y_{\omega} & \text { Discrete Fourier Transform of } y \text { at pulsation } \omega \\ v_{j} & \text { Window allowing to extract data from time segment } j \\ Y_{j, \omega} & \text { Discrete Fourier Transform of }\left(y \cdot v_{j}\right) \text { at pulsation } \omega \\ J & \text { Number of time segments } \\ \mathcal{S}_{x y}(\omega) & \text { Crossed-PSD matrix of signals } x \text { and } y \\ H(\omega) & \text { Transfer matrix }\end{array}$

\section{The modified Constitutive Relation Error in dynamics}

$\zeta^{2} \quad$ Constitutive Relation Error term

$r \quad$ Confidence into measurement coefficient

$\Pi \quad$ Frequency counterpart of projector $\Pi$

$\| \bullet \quad$ Hermitian model-to-data norm defined by the matrix $G_{y}$

$e_{j, \omega}^{2} \quad$ mCRE value at a given pulsation $\omega$ considering data from segment $j$

$D_{\omega} \quad$ Frequency bandwidth

$\Delta f \quad$ Frequency step

$z(\omega) \quad$ Frequency weighting function

$\mathcal{J}_{0} \quad$ mCRE normalization term

$\overline{\mathcal{J}} \quad$ Normalized mCRE functional

$\beta, \epsilon_{1}, \epsilon_{2} \quad$ Algorithm scalar thresholds

\section{Other mathematical operations and notations}

$\boldsymbol{t r}(\bullet) \quad$ Trace operator

-H Hermitian transpose

- $\quad$ Moore-Penrose pseudo inverse

E Mathematical expectation operator

- : $\square \quad$ Inner product between second-order tensors 


\section{Introduction}

\subsection{Context}

Control law synthesis and more generally dynamic systems stability and reliability assessment require the development of robust and predictive models. Those models can be constructed directly from measurements (black-box modeling) or derived after in-depth physical description of the phenomena involved (white-box modeling). In each case, a particular effort must be made to build those models and verify their domain of validity by experimental comparison.

Civil engineering problems are no exception to the need of deriving robust models for reliability assessment. In particular, in vibration-based damage detection or more generally structural health monitoring (SHM) applications, either parametrized (finite element) models or structured models (built from data-driven approaches) are used to follow the state of structures in operational or laboratory conditions [1,2].

For earthquake engineering purposes, in order to assess the vulnerability of civil engineering structures to seismic hazard, the CEA/TAMARIS facility carries out dedicated tests using shaking-tables moved by high-power hydraulic actuators on complete or partial structures at real or reduced scale [3, 4]. However, controlling the hydraulic actuators of the tables is still a challenging task, where robust and predictive models must be derived for enhanced performances.

Indeed, during seismic experiments on damaging specimens (such as reinforced-concrete constructions), the modal signature, which is the key input-feature for linear control, can face sudden changes. This imposes complex experimental protocols where test sequences of increasing level are carried out and the input-drive sent to the servo-actuators must be systematically corrected from one test to another to account for the observed frequency drop $[3,5]$. The ambition of this work is to make a first step towards the integration of numerical models in the shaking-tables control strategy, and to develop a model updating framework based on a offline/online update of the plant model for accurate control of the actuators. More precisely, a robust strategy for updating linear structural Finite Element (FE) models in low-frequency dynamics is described in this paper. A particular attention is paid for considering the wider possible range of measurements as experimental reference with a potentially low Signal-to-Noise Ratio (SNR) in order to broaden the scope of the proposed methodology.

\subsection{Model updating at a glance}

Model updating techniques aim at correcting parameters $p \in \mathcal{P}$ of a mathematical model $\mathcal{M}$ based on a set of observed outputs $(y)$ obtained under a given set of inputs $(e)$. Optimal parameters $\hat{p}$ are searched by minimizing a cost function $\mathcal{J}$ measuring the gap between measurements and their associated model predictions :

$$
\hat{p}=\arg \min _{p \in \mathcal{P}} \mathcal{J}(\mathcal{M}((e), p) ;(y))
$$

As all inverse problems, model updating problems are subjected to ill-posedness (in the Hadamard sense) and ill-conditioning issues especially because of measurement noise [6]. 
Regularization techniques must be considered for guaranteeing uniqueness of the solution and enforcing local ellipticity of the problem. Extensive literature reviews of validation/model updating approaches can be found [7-10]. The different methods described therein may be divided into two families as described below.

Deterministic methods (extensively reviewed in statics/quasi-statics in [9] and for nonlinear transient dynamics in [8]) build the cost function to be minimized as a distance from the response of the model to available measurements and overcome the ill-posedness issue using a so-called Tikhonov regularization term [11], containing a priori information $\mathcal{G}(p)$. The gap between model and data is measured using a (non-necessary Euclidean) norm \|\|$\bullet \|$ taking the entire amount of available data $((y),(e))$ into account. The cost function reads:

$$
\mathcal{J}(p)=\|\Pi \circ \mathcal{M}((e), p)-(y)\|^{2}+\alpha \mathcal{G}(p)
$$

where $\Pi$ is a projection operator, whose non-zero terms allow to extract model predictions at sensors location in order to compare them with the measured quantities, leading to the data-to-model distance ( $\Pi \circ \mathcal{M}(e(t), p)-y(t)$ ) (also called prediction error). The weighting coefficient $\alpha$ associated to the regularization term $\mathcal{G}(p)$ can be optimally calibrated using various techniques such as the Morozov discrepancy principle [12], the L-curve method [13] or the Arcangeli principle [14].

Alternatively to direct data-to-model comparison (2), experimental modal features can be used as a reference for dynamics problems: the data-to-model distance then directly compares experimental eigenfrequencies, modeshapes and damping ratios to those predicted by the FE model. A modal analysis step is often performed in an experimental or operational context to extract the reference modal signature (see [15] for stochastic subspace identification in the time domain, [16] for frequency-domain techniques and [17] for a complete review). Such features can take into account damage evolution, thermal effects or other environmental effects in a long-time monitoring context. Sensitivity analysis methods, reviewed in [18-20] and applied in [21-23] among many other works, are then carried out for localizing erroneous areas and correcting associated physical parameters.

Stochastic methods, in turn, use the Bayesian inference framework to describe uncertainties on the parameter estimation $[6,10,24]$. The Bayesian approach aims at improving the prior knowledge on the parameters probability density function (pdf) denoted using both experimental data $(y)$ and the prediction model $\mathcal{M}$. The updating strategy is based on the eponymous Bayes theorem, which builts the posterior pdf from the prior pdf and the likelihood function. The prior pdf is generally chosen based on engineering judgment and describes an initial guess of the parameters distribution in the absence of observations. The likelihood function can be interpreted as a measure of how good the set of parameters $p$ applied to model $\mathcal{M}$ succeeds in explaining the observations $(y)$. Optimal parameters $\hat{p}$ can then be recovered based on classical Maximum Likelihood Estimation (MLE) principle, i.e. such as maximizing the log of the likelihood function.

\subsection{The modified Constitutive Relation Error ( $m C R E$ )}

In this contribution, since low-SNR measurements may lack of richness to update FE models properly, a cost function with enriched physical meaning and strong mechanical con- 
tent is chosen. Following the strategy proposed by Ladevèze and co-workers, a deterministic energy residual is defined as a quadratic model-to-measurements distance written in the frequency domain and enriched with a term based on the concept of Constitutive Relation Error (CRE) [25]. The idea behind this enrichment is to improve the ellipticity properties of the cost function by adding a term which relaxes unreliable parts of the model. Introduced in the 1980s for the purpose of FE verification (and extensively developed in [26-32] among many other references), the CRE concept was later adapted for model validation [33-37] in structural dynamics to define a modified CRE (mCRE) residual to be minimized with respect to the set of updated parameters. The mCRE functional reads:

$$
\operatorname{mCRE}(p)=\operatorname{CRE}(p)+\frac{r}{1-r}\|\| \Pi \circ(\mathcal{M}((e), p))-(y)\|\|^{2}
$$

with a scalar $r$ for balancing the two terms. This energy-based residual offers interesting advantages. It improves local convexity properties compared to classical least-square functionals [9, 38-40]. Besides, the CRE part of the residual, computed over the whole structure, allows to select the most erroneous areas in order to restrain the updating process to a few parameters; this provides another additional regularization (in the Tikhonov sense), particularly when the number of parameters to update becomes important. The mCRE model updating methodology has proved robustness and efficiency through a large number of applications involving defect detection [41-44], very noisy or even corrupted measurements [38, 45-47], tolerance to incomplete boundary conditions [40, 48, 49], full-field material identification [50-52], acoustics [53, 54], wall-slab RC joint characterization [55], real-time model updating and data-assimilation [56] or coupling with model order reduction techniques like Proper Generalized Decomposition (PGD) [57]. Recently, a unified formulation of the mCRE has been proposed (in the time domain) for the full updating of constitutive relations and evolution laws in a nonlinear context [58].

Contrary to modal analysis techniques, the mCRE-based model updating algorithm is not restrained to the processing of linear responses of structures. However, the knowledge of the input signal is mandatory for the mCRE-based model updating methodology proposed herein, contrary to operational modal analysis techniques applied to SHM that only take advantage of output-only measurements. Besides, the explicit evaluation of a local model error indicator, namely the CRE part of the residual, provides more insight on the validity of the model itself compared to classical finite element model updating methods.

In this paper, a robust and automated mCRE-based model updating framework is derived for considering low-SNR random processes as experimental reference. A particular attention is paid to the weighting of the two terms defining the cost function to minimize (CRE residual and data-to-model distance). Indeed, a preliminary numerical study (also presented in this contribution) highlighted that an inappropriate choice of the weighting factor $r$ could lead to irrelevant results, especially when considering low-SNR measurements. A first contribution is proposed for automatically tuning the weighting factor $r$ based on the L-curve principle, bringing the overall methodology more robustness with respect to measurement noise. However, the mCRE-based model updating algorithm in its initial frequency version may still fail at identifying erroneous parameters or finding relevant optimal values, especially when considering low-SNR datasets. A second improvement is then proposed in such situations 
under classical ergodicity conditions with an extension of the model updating strategy based on $(i)$ a preliminary PSD-type data preprocessing, $(i i)$ an automated emphasis on the experimental eigenfrequencies, and (iii) a new formulation of the mCRE-based cost function that guarantees convergence of data-to-model distance in terms of PSDs. The efficiency of those improvements is illustrated using both synthetic measurements and actual test results acquired during the SMART2013 shaking-table test-campaign during which a RC specimen was submitted to a sequence of increasingly damaging ground motion tests. In these numerical applications, a linear "equivalent" updated model weighted by a restrained number of parameters has been able to track the overall structural state and eigenfrequencies, which are of major concern for adaptive control design prospects.

The paper is structured as follows. Section 2 presents the fundamentals of the proposed mCRE-based model updating framework and recalls the reference problem to be solved. The associated cost function is introduced and the way random processes are dealt with for ensuring good accordance between data and corrected model predictions in terms of PSD is explained, with particular attention on how the proposed choice of $\| \bullet \bullet||$ defines a norm on the PSD sequence of the prediction error. Section 3 gives more insights into the minimization algorithm itself. Technical details on different ingredients such as modal projection, control of the procedure, and choice of the parameters to update are explicitly given. Section 4 presents the results of the two numerical applications processed in this work. The first earthquake-engineering-inspired academic example (plane frame submitted to Gaussian acceleration loading) is first presented and enables a full discussion on the efficiency and limits of the methodology with respect to measurement noise of known level. The results obtained processing the SMART2013 database are then detailed: after a short contextualization and description of the FE model borrowed from [3], updated parameters are given for the whole set of testings. In both cases, particular attention is paid to the tuning of the weighting factors of the algorithm with respect to measurements. The efficiency of the methodology is particularly illustrated by the good correlation between the modal signature produced by the corrected FE model and the modal identification results formerly obtained using datadriven subspace-based algorithms [5]. Insights are also provided regarding the (appropriate) choice of parameterization for optimal model updating. Conclusions and prospects are finally drawn in Section 5.

\section{Model updating framework based on the constitutive relation error}

\subsection{The reference problem}

Let one consider a linear structure, within a domain $\Omega$, at time $t \in[0 ; T]$ as shown in FIG. 1. This structure is subjected to a given time-dependent displacement field $u_{d}$ on the boundary $\partial_{1} \Omega \subset \partial \Omega$ and to surface and body loads, respectively denoted $F_{d}$ and $f_{d}$, applied on $\partial_{2} \Omega \subset \partial \Omega$ and $\Omega$, with $\partial_{1} \Omega \cup \partial_{2} \Omega=\partial \Omega$ and $\overline{\partial_{1} \Omega \cap \partial_{2} \Omega}=\emptyset$.

The reference problem consists in finding, for all $M \in \Omega$ and $t \in[0 ; T]$, the displacement field $u(M, t) \in \mathcal{U}^{[0 ; T]}$ and the stress field $\sigma(M, t) \in \mathcal{S}^{[0 ; T]}$ verifying: 


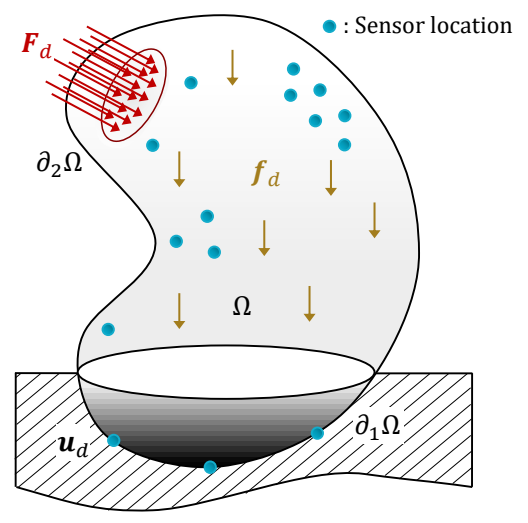

FiguRE 1: The structure and its environment.

$$
\begin{aligned}
\text { Initial conditions } & \forall M, \quad u\left(M, t_{0}\right)=u_{0}, \quad \dot{u}\left(M, t_{0}\right)=\dot{u}_{0} \\
\text { Kinematic boundary conditions } & \forall M \in \partial_{1} \Omega, \forall t, \quad u(M, t)=u_{d}(M, t) \\
\text { Equilibrium equations } & \forall M \in \partial_{2} \Omega, \quad \forall t, \quad \sigma(M, t) \cdot n=F_{d}(M, t) \\
& \forall(M, t), \quad \rho \frac{\partial^{2} u(M, t)}{\partial t^{2}}=\nabla \cdot \sigma+f_{d} \\
\text { Constitutive relation } & \forall(M, t), \quad \sigma(M, t)=\mathcal{K}: \varepsilon(u(M, t))+\mathcal{D}: \dot{\varepsilon}(u(M, t))
\end{aligned}
$$

with $\varepsilon(\bullet)=\frac{1}{2}\left(\nabla \bullet+\nabla^{T} \bullet\right)$ the linearized strain tensor, and $\mathcal{K}, \mathcal{D}$ the fourth-order Hooke and dissipation tensors, respectively. Consequently, the stress $\sigma$ can be divided in an elastic part $\sigma^{e}$ and a dissipative part $\sigma^{d}$ such that $\sigma(M, t)=\sigma^{e}+\sigma^{d}$.

The spaces where the solution fields are searched are said kinematically admissible for $\mathcal{U}^{[0 ; T]}$ and dynamically admissible for $\mathcal{S}^{[0 ; T]} \cdot \mathcal{U}^{[0 ; T]}$ is defined as the set of vectors such that for all $t, u(t) \in H^{1}(\Omega)$ verifying the kinematic boundary conditions, whereas $\mathcal{S}^{[0 ; T]}$ is the set of symmetric second-order tensors $\sigma(t) \in L^{2}(\Omega)(\forall t)$ verifying the equilibrium equations. This (direct) problem is well-posed in the Hadamard sense i.e. the solution exists, is unique and stable with respect to its input parameters.

\subsection{Measurements in the frequency domain}

Let one consider a set of sensors scattered over the structure as illustrated in Fig. 1. Sensing devices classically acquire several types of measurements generally associated to the unknown displacement field $u$ or its time and space derivatives, although excitation forces or actuator state variables may also be available. In practice, the dataset of observed outputs $(y)$ may contain any physical measured quantity deriving from a displacement field at sensors location (displacements, velocities, accelerations, strain, etc.), whereas inputs (e), if measured, are associated to the loading (imposed displacements, forces or accelerations). Besides, in the context of low-frequency dynamics, the following model updating strategy will be written in the frequency domain, so that the frequency complex counterparts of inputs and outputs are introduced and denoted $(E(\omega))$ and $(Y(\omega))$ for all pulsation $\omega$, respectively. 
Taking the measurements as additionnal boundary conditions overspecifies the above mechanical reference problem, that becomes ill-posed. Indeed, one shall remark that, from an experimental point of view, the knowledge of the frequency complex amplitudes of inputs and outputs $(E(\omega))$ and $(Y(\omega))$ is subjected to uncertainties because of measurement noise, finite nature of the recordings, sampling process, sensors offset or miscalibration, anti-aliasing filters, etc. Furthermore, when studying systems submitted to stochastic inputs, random signals, viewed as discrete-time sequences, do not have finite energy and hence do not possess a discrete-time Fourier transform (see e.g. [59]). However, they usually have a finite average Power Spectral Density (PSD). Under stationarity assumption, and introducing a unit window $v$ of length $T$ such that $x_{T}(t)=v \cdot x(t)$, the PSD matrix of a random signal $x(t)$, denoted $\mathcal{S}_{x x}(\omega)$, can be defined for all $\omega$ as:

$$
\mathcal{S}_{x x}(\omega) \triangleq \lim _{T \rightarrow+\infty} \mathbf{E}\left(\frac{1}{T} \int_{-\infty}^{+\infty}\left[X_{T}(\omega)\right]\left[X_{T}(\omega)\right]^{H} d \omega\right)
$$

where $X_{T}$ is the Fourier transform of $x_{T}$, and $\mathbf{E}()$ and $\bullet^{H}$ denote the mathematical expectation and Hermitian transpose operators, respectively. Defining in practice a correct windowing (number of segments, type and length of the windows for apodization, zeropadding, etc.) might not be an easy task and could add uncertainty to a quantity (the PSD matrix) that, by nature, can only be estimated in a statistical manner.

An example of the windowing used for the SMART2013 application can be seen in FIG. 2. Let us simply assume here for the sake of generality that when dealing with random processes, a given set of $J$ possibly overlapping windows $\left\{v_{j}\right\}_{j=1}^{J}$ is used for the computation of the PSD matrices. Once applied to the input and output time-series, this windowing step provides an ensemble of complex values denoted $\left(E_{j, \omega}\right)$ and $\left(Y_{j, \omega}\right)$.

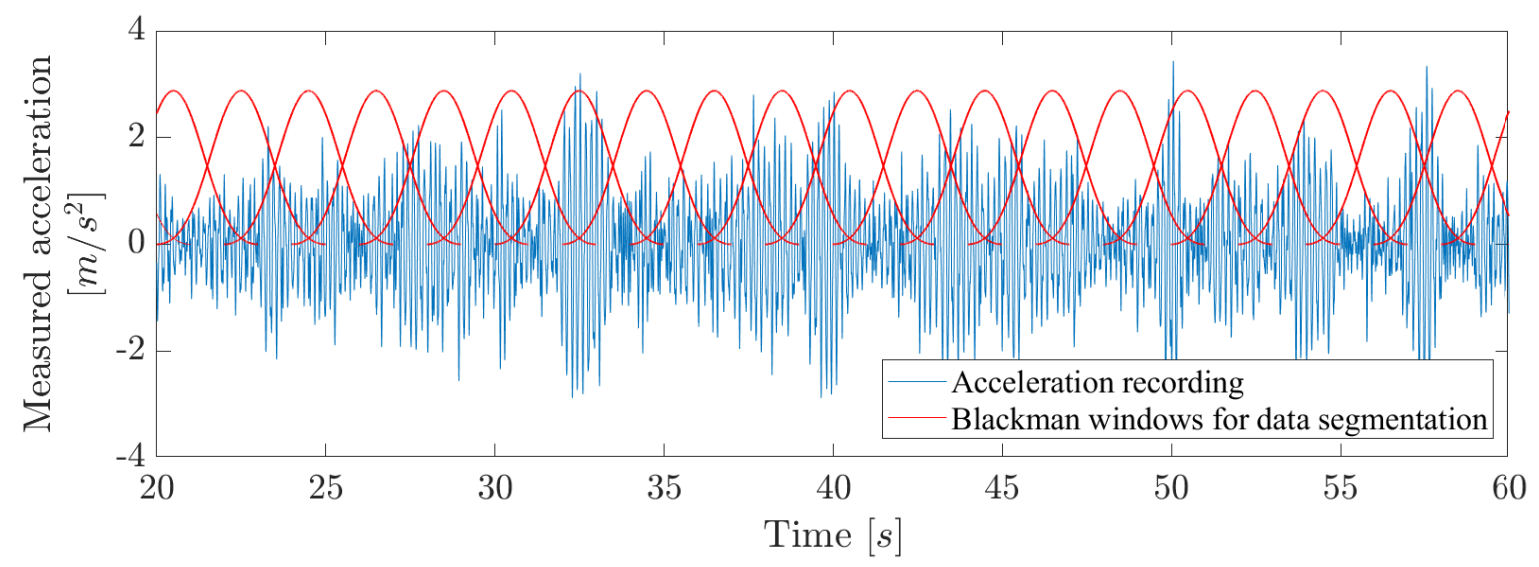

FIGURE 2: Acceleration time histories measured during the SMART2013 shaking-table testing campaign (Run \#6) and 60\%-overlapping Blackman windows defined for computing the PSD matrix of the measured outputs.

\subsection{Model updating problem including both CRE and measurements}

This section explains how the cost function to minimize is constructed from the less reliable data and equations of the reference problem. The complete problem is presented here 
assuming random inputs and thus some data windowing. Note that the classical deterministic mCRE-based model updating problem found in former literature works can be recovered as a particular case where only one unitary window is processed.

The key point here consists in splitting the data and equations that are considered as reliable from those considered as unreliable. TAB. 1 presents the separation made for the present study. The CRE construction lies into the distinction between reliable and unreliable quantities when considering the whole set of equations defining the reference mechanical problem. This separation is non-unique and deeply relies on the case study: for instance, the loading could be considered as unreliable with the rest of the measurements (see [37] for an example of such an application and [60] for a complete discussion on the possibility and limitations of considering the loading as fully unknown).

\begin{tabular}{|c|c|c|}
\hline & Reliable & Unreliable \\
\hline Model and eqs. & $\begin{array}{l}\text { - Geometry } \\
\text { - Kinematic boundary conditions (5) } \\
\text { - Equilibrium equations }(6-7) \\
\text { - Dissipative part of the constitutive } \\
\text { relations (8) }\end{array}$ & $\begin{array}{l}\text { - Elastic part of the constitutive } \\
\text { relations }(8)\end{array}$ \\
\hline Experimental data & $\begin{array}{l}\text { - Identified pulsations } \omega \\
\text { - Sensors position and orientation } \\
\text { - Measured inputs }(e) \rightarrow\left(E_{j, \omega}\right)\end{array}$ & - Measured outputs $(y) \rightarrow\left(Y_{j, \omega}\right)$ \\
\hline
\end{tabular}

TABLE 1: Distinction between reliable and unreliable information on a single segment $j$ at pulsation $\omega$ for the considered problem.

A solution $s=\left(u, \sigma^{e}, \sigma^{d}\right)$ verifying the reliable equations is then declared admissible. Assuming that the system is studied on a single segment $j$ at pulsation $\omega$, the corresponding admissibility space will be denoted $\mathcal{S}_{j, \omega}^{a d}$ in the following. As doubt is put on the constitutive relations, the energetic norm of the distance between the kinematically and dynamically admissibility spaces defines a measure of how consistent the solution $s=\left(u, \sigma^{e}, \sigma^{d}\right)$ is with respect to the mechanical problem. Therefore, the degree of non-verification of the (assumed unreliable) constitutive relations defines the CRE, or model error, denoted $\zeta^{2}$ and defined as a linear combination of two terms:

- the CRE associated with the elastic behavior:

$$
\zeta_{\text {elas }}^{2}(s)=\frac{1}{2} \int_{\Omega}\left(\sigma^{e}-\mathcal{K}: \varepsilon(u)\right): \mathcal{K}^{-1}:\left(\sigma^{e}-\mathcal{K}: \varepsilon(u)\right) \mathrm{d} \Omega
$$

- the dissipation error:

$$
\zeta_{\text {diss }}^{2}(s)=\frac{1}{2} \int_{\Omega}\left(\sigma^{d}-\mathcal{D}: \dot{\varepsilon}(u)\right): \mathcal{D}^{-1}:\left(\sigma^{d}-\mathcal{D}: \dot{\varepsilon}(u)\right) \mathrm{d} \Omega
$$

In this work, according to TAB. 1 , it is chosen to update only structural stiffness properties, meaning that only the elastic part of the constitutive relations is assumed subject 
to caution. Practically, $\zeta^{2}=\zeta_{\text {elas }}^{2}$ in the following. Indeed, for correcting FRFs in lowfrequency dynamics, a current practice consists in updating stiffness parametrization first to update eigenfrequencies, before updating damping parameters for correcting the narrowness of the resonant peaks in a second step. Indeed, the correction of frequency response functions consists in a two-stage scheme where stiffness properties are firstly updated alone for correcting eigenfrequencies. Damping properties are updated in a second stage to correct the narrowness of the resonant peaks. Although only stiffness properties are updated in this work, please note that an analogous formulation can be written when casting doubt on the dissipative part of the constitutive relations, the mCRE being a well-suited functional for updating damping properties too [35].

In the model identification framework, the extension of the concept of energy residual from the CRE on the less reliable measurements leads to the so-called modified Constitutive Relation Error (mCRE) written as

$$
\forall j, \forall \omega \quad e_{j, \omega}^{2}(s) \triangleq \zeta_{j, \omega}^{2}(s)+\frac{r}{1-r}\left\|\Pi U_{j, \omega}-Y_{j, \omega}\right\|^{2}
$$

This energy-based residual is composed of two terms: the CRE itself $\zeta_{j, \omega}^{2}(s)$ measuring the degree of non-verification of the elastic part of the constitutive relations and a model-to-data distance from the predictions $U_{j, \omega}$ to the measurements $Y_{j, \omega}$.

The projection operator $\Pi$ is the frequency counterpart of $\Pi$, previously introduced in (2). As measurements derive from the displacement field and its time derivatives, the nonzero coefficients are integer powers of $i \omega$ for extracting the measured components of $U_{j, \omega}$ at corresponding sensor positions and directions. The Hermitian norm involved in the distanceto-measurement term is defined such that $\|\bullet\|^{2}=\frac{1}{2}(\bullet)^{H}\left[G_{y}\right](\bullet)$ using a symmetric positivedefinite matrix $G_{y}$ of appropriate dimension. The choice of $G_{y}$ is not critical, however special care should be taken for guaranteeing that $\|\bullet\|^{2}$ is homogeneous to $\zeta^{2}$ and equivalent in level. In this work, $G_{y}$ is constructed from the initial stiffness operator condensed on the sensors locations using classical Guyan reduction [35, 61]. The tuning factor $r \in] 0$; 1 [ enables one to give more or less confidence to the measurements; close-to-unit values can be specified when measurements are considered reliable whereas close-to-zero values concern more corrupted recordings or having higher noise level. The choice of this tuning parameter is usually set to a default value of $r=0.5$ in many applications [34-37, 46, 55, 62] with satisfactory results. The sensitivity of $r$ with respect to the measurements was already illustrated in [36], with the conclusion that $r=0.5$ is a convenient value for most cases.

In summary, on each segment $j$ and each pulsation $\omega$, a solution living in the admissibility space $\mathcal{S}_{j, \omega}^{a d}$ and verifying the less reliable data and equations as closely as possible is searched. The reformulated problem thus reads as a constrained minimization problem $\left(P_{j, \omega}\right)$ that consists in seeking a solution $\hat{s}$ such that:

$$
\left(P_{j, \omega}\right) \quad \hat{s}(p)=\arg \min _{s \in \mathcal{S}_{j, \omega}^{a d}} e_{j, \omega}^{2}(s ; p)
$$

Once the solution $\hat{s}(p)$ of $\left(P_{j, \omega}\right)$ is computed for each couple $(j, \omega)$, the value $e_{j, \omega}^{2}(\hat{s}(p) ; p)$ 
can be evaluated and the final cost function $\mathcal{J}$ to be minimized with respect to parameters $p$ reads:

$$
\mathcal{J}(p) \triangleq \int_{D_{\omega}} z(\omega) \underset{j}{\mathbf{E}}\left(e_{j, \omega}^{2}(\hat{s}(p) ; p) \mathrm{d} \omega\right)
$$

where $z(\omega)$ is a positive frequency weighting function defined over the frequency range $D_{\omega}$ which verifies $\int_{D_{\omega}} z(\omega) d \omega=1$. The complete model updating problem $(P)$ finally reads:

$$
\hat{p}=\arg \min _{p \in \mathcal{P}} \mathcal{J}(p)
$$

To highlight the strong nesting between problems $\left(P_{j, \omega}\right)$ and $(P)$ (due to the dependency of the behavior operators into the set of parameters), the overall problem can be reformulated as follows:

$$
\hat{p}=\arg \min _{p \in \mathcal{P}} \int_{D_{\omega}} z(\omega) \underset{j}{\mathbf{E}}\left(\min _{s \in \mathcal{S}_{j, \omega}^{a d}} e_{j, \omega}^{2}(s(p) ; p) \mathrm{d} \omega\right)
$$

\subsection{Contributions for enhanced robustness with respect to ergodicity and measurement noise}

In this work, a notable effort has been made for proposing automated tuning procedures which enhance robustness of the mCRE-based model updating strategy with respect the following aspects:

- Enhanced robustness with respect to measurement noise - An automated tuning procedure of the confidence into measurements coefficient $r$ that naturally discriminates low-SNR measurements is proposed herein. Indeed, the choice of $r$ is crucial for providing relevant parameter estimates, as will be illustrated in Section 4. Besides, as shown in Fig. 7, a unitary frequency weighting function $\left(z(\omega)=1 /\left\|D_{\omega}\right\| \forall \omega\right)$ leads to a monotonic evolution of the mCRE (seen as a function of $\omega$ ). Mostly because of the (non-tempered) high values in the low-frequency range, the procedure may fail at providing relevant identification results. A frequency weighting function based on the frequency content of the considered measurements allows to automatically favor the vicinity of the experimental modal eigenfrequencies, improving the sensitivity of parameters and the quality of the provided estimates.

- Enhanced robustness with respect to low-magnitude ergodic inputs - during shaking table experiments, non-damaging broad-band ergodic tests are performed to identify modal signature changes. Similarly to what is classically done for processing periodograms for random processes, a data windowing extension of the mCRE functional has been shown effective for preventing potential divergence of the model updating algorithm and providing more stable estimates even when considering low-SNR measurements.

Complete explanations about these contributions are given in the remainder of this section. 


\subsubsection{L-curve methodology for an optimal confidence into the measurements}

When processing low-SNR random measurements, the corrections provided by the algorithm with a default value $r=0.5$ may lead to inconsistent results (see applications in Section 4). To circumvent this lack of robustness, an automated selection based on the Lcurve principle [13] is proposed in this paper to define an optimal value of $r$ with respect to measurement noise and in accordance with the a priori confidence one has into the quality of the measurements. The L-curve principle states that the optimal value of $r$ balances the model and measurement error, in the following manner:

$$
\hat{r}=\arg \min _{r \in[0 ; 1[}\left\{|\underbrace{\int_{D_{\omega}}^{\mathbf{E}}\left(\zeta_{j, \omega}^{2}(\hat{s})\right) \mathrm{d} \omega}_{\text {Model error }}-\underbrace{\int_{D_{\omega}}^{\mathbf{E}}\left(\frac{r}{1-r}\left\|\Pi \hat{U}_{j, \omega}-Y_{j, \omega}\right\|^{2}\right) \mathrm{d} \omega}_{\text {Measurement error }}|\right\}
$$

A pedagogical illustration of this procedure is available in Section 4.1.2 - FIG. 6.

\subsubsection{Frequency bandwidth, frequency weighting}

The model updating procedure is conducted on a given frequency bandwidth $D_{\omega}=$ $\left[\omega_{\min } ; \omega_{\max }\right]$ which contains the essential part of the mechanical energy of the system. For seismic applications, ground motions usually have a significant frequency content up to $50 \mathrm{~Hz}$, which implies that $D_{\omega} \subset[0 \mathrm{~Hz} ; 50 \mathrm{~Hz}]$. Besides, one should note that the frequency weighting function $z(\omega)$ can be used as a modulation function to favor frequencies deemed to have the largest influence on the model updating procedure. In this work, we propose an automated computation of $z(\omega)$ from experimental datasets, based on the fact that, for low-frequency dynamics, the essential frequency content of the measurements is gathered around experimental natural frequencies. In this work, a normalized version of the Complex Mode Indicator Function (CMIF) [63] on transfer functions is used for frequency weighting as explained in the following lines. One first needs to compute the transfer matrix $H(\omega)$ from the crossed input/output PSD matrices $\left[\left[\mathcal{S}_{y y}(\omega) \mathcal{S}_{y e}(\omega)\right] ;\left[\mathcal{S}_{e y}(\omega) \mathcal{S}_{e e}(\omega)\right]\right]$ using one of the three following formulas:

$$
\begin{aligned}
H(\omega) & =\left[\mathcal{S}_{y y}(\omega)\right]\left[\mathcal{S}_{e y}(\omega)\right]^{\dagger} \\
H(\omega) & =\left[\mathcal{S}_{y e}(\omega)\right]\left[\mathcal{S}_{e e}(\omega)\right]^{\dagger} \\
H(\omega) & =\left[\mathcal{S}_{y y}(\omega) \mathcal{S}_{y e}(\omega)\right]\left[\mathcal{S}_{e y}(\omega) \mathcal{S}_{e e}(\omega)\right]^{\dagger}
\end{aligned}
$$

where $\bullet^{\dagger}$ refers to the Moore-Penrose pseudo-inverse. The dominant singular value $\Sigma_{1}(\omega)$ of matrix $H(\omega)$ has the property of peaking in the vicinity of natural frequencies and can be advantageously used for defining $z(\omega)$. This indicator based on the transfer matrix $H$ is called $H-\mathrm{CMIF}$ in what follows (owing to its similarities with classical CMIF). The preliminary smoothing of the PSDs is therefore crucial for allowing the detection of resonant peaks without misinterpretation due to the random nature of the signals. The efficiency of this $H$-CMIF-based frequency weighting function is highlighted in the following numerical 
applications in Section 4, where some examples of $H$-CMIF used as weighting function are shown (see Fig. 7 or 13).

In order to fulfill the condition $\int_{D_{\omega}} z(\omega) \mathrm{d} \omega=1$, the $H$-CMIF weighting function $z(\omega)$ is derived as

$$
z(\omega)=\frac{\Sigma_{1}(\omega)}{\int_{D_{\omega}} \Sigma_{1}(\omega) \mathrm{d} \omega}
$$

\subsubsection{Convergence to measurements in terms of PSDs}

In the perspective of dealing with random processes, our claimed objective is to build a cost function that, once minimized with respect to the model parameters $p$, would make the model predictions converge to the measurements in terms of PSD. One could legitimately ask if this desired requirement is met. Let us prove this claim and consider the integrated and averaged model-to-measurements term, that reads:

$$
\|\Pi \circ(u)-(y)\|^{2}=\int_{D_{\omega}} z(\omega) \underset{j}{\mathbf{E}}\left(\left\|\Pi U_{j, \omega}-Y_{j, \omega}\right\|^{2}\right) \mathrm{d} \omega
$$

where the integral sign and mathematical expectation can be permuted if the realizations on each segment $j$ are statically decorrelated. As previously explained, the involved norm is written using real symmetric positive-definite matrix $G_{y}$. Let thus introduce the predictionerror sequence $(\eta)=\left(G_{y}^{1 / 2}(\Pi u-y)\right)$ using the square root of matrix $G_{y}$. The distance term reads:

$$
\forall j, \forall \omega \quad\left\|\Pi U_{j, \omega}-Y_{j, \omega}\right\|^{2}=\frac{1}{2}\left(\Pi U_{j, \omega}-Y_{j, \omega}\right)^{H}\left[G_{y}\right]\left(\Pi U_{j, \omega}-Y_{j, \omega}\right)
$$

Now taking the mathematical expectation of this last expression yields:

$$
\forall \omega \quad \underset{j}{\mathbf{E}}\left(\left\|\Pi U_{j, \omega}-Y_{j, \omega}\right\|^{2}\right)=\operatorname{tr}\left(\mathcal{S}_{\eta \eta}(\omega)\right) \geqslant 0
$$

by definition of the PSD sequence, where $\operatorname{tr}(\bullet)$ refers to the trace operator. Thus, if the matrix $G_{y}$ is symmetric, positive and definite, the following distance to measurements term:

$$
\|\Pi \circ(u)-(y)\|^{2}=\int_{D_{\omega}} z(\omega) \operatorname{tr}\left(\mathcal{S}_{\eta \eta}(\omega)\right) \mathrm{d} \omega
$$

obviously defines a norm on the PSD sequence $\left(\mathcal{S}_{\eta \eta}(\omega)\right)_{\omega \in D_{\omega}}$ which is exactly the property that was looked for. Minimizing a weighted norm of the prediction error PSD matrix on the whole frequency domain $D_{\omega}$ with respect to $p$ thus makes the model predictions converge to the measurements.

Therefore, the preliminary data windowing and the extended averaging definition of the model updating enable a better integration of random processes and bring an additional statistical stability of the parameters with respect to measurement noise, as illustrated in the 
numerical applications in Section 4. Let us finally recall that the deterministic methodology described in [34-36] can be recovered using a single segment with a single unitary rectangular window.

\section{Implementation of the model updating algorithm}

As presented in the previous section, the mCRE-based model updating algorithm implies the solution of two nested minimization problems. This section assumes FE discretization of the continuous problem and details the solution scheme of $\left(P_{j, \omega}\right)$ and $(P)$ when optimal stiffness parameters are sought in $\mathcal{P}$. A general pseudo-code of the complete procedure is also given at the end of this section (see ALG. 1). In the following, the $(j, \omega)$ subscripts are omitted fo the sake of clarity as long as there is no ambiguity.

\subsection{Solution of the problem $\left(P_{j, \omega}\right)$}

In a $\mathrm{FE}$ framework, the discretization of the reference problem leads to the assembly of stiffness, damping and mass FE matrices, respectively denoted $K, D, M$. Likewise, any vector describing mechanical states of the system is discretized by a vector of nodal values: the classical kinematically admissible nodal field $U$ and an auxiliary dynamically admissible nodal displacement field $V$ are thus introduced. $V$ is associated to the discretized stress tensor through a change of variables and verifying (in a FE sense) the constitutive relations (8) and the dynamic equilibrium (7). The constitutive relation error $\zeta^{2}$ measures the reciprocity gap between the two unknown nodal displacement fields $U$ and $V$.

In this contribution, in the perspective of interpreting local stiffness variations as damage pattern, the constitutive relation error $\zeta^{2}$ term involved in the definition of the cost function (12) will only involve the elastic part of the constitutive relations. The discrete mCRE functional thus becomes:

$$
e^{2}(U, V, p)=\frac{1}{2}(U-V)^{H} K(p)(U-V)+\frac{1}{2} \frac{r}{1-r}(\Pi U-Y)^{H} G_{y}(\Pi U-Y)
$$

where one can notice that the stiffness matrix $K(p)$ explicitly depends on the parameters contained in $p$. The solution fields $(U, V) \in \mathcal{S}^{a d}$ must satisfy dynamical equilibrium, i.e.

$$
-\omega^{2} M U+i \omega D U+K(p) V=F
$$

The minimization of the mCRE (26) under dynamic equilibrium condition (27) is performed using a classical Lagrange multiplier vector $\Lambda$ and the augmented cost function reads:

$$
\mathcal{L}(U, V, \Lambda, p)=e^{2}(U, V, p)+\Lambda^{H}\left[\left[-\omega^{2} M+i \omega D\right] U+K(p) V-F\right]
$$

Thus, the stationarity of $\mathcal{L}$ with respect to the fields $(U, V, \Lambda)$ leads to the solution of a linear system: 


$$
A\left\{\begin{array}{c}
\hat{\Lambda} \\
\hat{U}
\end{array}\right\}=b \quad \text { with }\left\{\begin{array}{c}
A=\left[\begin{array}{cc}
{\left[K(p)+\imath \omega D-\omega^{2} M\right]^{H}} & \frac{r}{1-r} \Pi^{H} G_{y} \Pi \\
-K^{H}(p) & {\left[K(p)+\imath \omega D-\omega^{2} M\right]}
\end{array}\right] \\
b=\left\{\begin{array}{c}
\frac{r}{1-r} \Pi^{H} G_{y} Y \\
F
\end{array}\right\}
\end{array}\right.
$$

and $\hat{\Lambda}=\hat{U}-\hat{V}$. The direct solution of the system (29) can be very costly in terms of CPU resources. Indeed, the matrix $A$ is of consequent size $2 N_{d} \times 2 N_{d}\left(N_{d}\right.$ being the number of dofs) and $\left(P_{j, \omega}\right)$ must be solved for all couples $(j, \omega)$ each time the solution for a given parameter set $p$ has to be evaluated. A projection on a truncated modal basis made of the $L$ first eigenmodes is used to reduce the size and thus the computational cost of the solution of (29). An appropriate choice for $L$ can be made such that $\omega_{L} \gg \max \left(D_{\omega}\right)$ and $L \ll N_{d}$ (see [35] for additional details). In practice, a sufficiently rich and constant basis computed in preamble of the model updating algorithm enables to avoid a complex dependency of the solution fields $(\hat{U}, \hat{V})$ into the parameters.

\subsection{Solution of the problem $(P)$}

Optimal parameters $\hat{p}$ are obtained recursively as the limit of the sequence $\left(p^{(k)}\right)_{k}$ in an iterative localization/correction algorithm for which an overall pseudo-code is given in ALG. 1 and key ingredients are detailed below.

The nonlinear minimization problem $(P)$ is solved using a classical BFGS minimization algorithm [64-67] with supplied analytical gradient (the interested reader is refered to [37] for a complete demonstration).

\subsubsection{Description of the parameter space $\mathcal{P}$}

Because a sparse distribution of sensors is available and because one intends to provide global corrections, a piecewise constant weighting of the Young modulus defined on a nonoverlapping subdomain decomposition of the structure $\Omega=\cup_{n=1}^{N} \Omega_{n}$ is used. In practice, the updated stiffness FE model reads:

$$
K(p)=\bigoplus_{n=1}^{N} p_{n} K_{n, 0}, \quad p_{n}>0 \forall n
$$

with $\bigoplus$ referring to the $\mathrm{FE}$ assembly procedure and $K_{n, 0}$ to the initial stiffness matrix of subdomain $n$. In order to keep physical meaning, each parameter must satisfy $p_{n}>0$. They can characterize any physical property that contributes globally to the FE stiffness matrix. The subdomains are user-defined in the preliminary step of the model updating algorithm and allow to reduce the number of updated parameters, which contributes to limit the illposedness of the updating problem (see Section 4.2.4 for additional details). Note that the direct weighting of FE stiffness submatrices allows to identify structural damage as stiffness loss. This choice is based on the will to use a linear "equivalent" model for identifying modal signature changes. 


\subsubsection{Localization of the most erroneous areas}

At each iteration $k$, only a small amount of parameters is corrected. These parameters are chosen based on the localization of the most erroneous subdomains (in the CRE sense). Indeed, the nature of the FE operators allows to extract the CRE part of the cost function per subdomain $\zeta_{n}^{2}$ for $n \in \llbracket 1 ; N \rrbracket$. Using a threshold $\beta \in[0 ; 1]$, one can determine if a given subdomain $n$ belongs to the most erroneous ones in the structure using the following criterion

$$
\zeta_{n}^{2} \triangleq \frac{1}{2}\left(U_{n}-V_{n}\right)^{H} K_{n}(p)\left(U_{n}-V_{n}\right) \geqslant \beta \max _{n \in \llbracket 1 ; N \rrbracket}\left(\zeta_{n}^{2}\right)
$$

with, e.g. $\beta=0.8$. This guarantees that a restrained number of parameters is updated at each correction step and brings regularization in the Tikhonov sense.

\subsubsection{Monitoring of the algorithm - convergence criteria, computational effort}

The FE mesh density and the size of the structure strongly depend on the studied problem, and therefore have much incidence on the mCRE numerical value. In order to robustly assess the convergence of the method, a normalization term is defined at the first iteration of the algorithm, leading to a normalized cost function $\overline{\mathcal{J}}$ :

$$
\begin{gathered}
\overline{\mathcal{J}}(p)=\frac{\mathcal{J}(p)}{\mathcal{J}_{0}} \\
\text { with } \mathcal{J}_{0}=\sum_{j \in \llbracket 1 ; J \rrbracket} \sum_{\omega \in D_{\omega}}\left[\frac{1}{2}\left(U^{(0)}+V^{(0)}\right)^{H} K\left(p^{(0)}\right)\left(U^{(0)}+V^{(0)}\right)\right]
\end{gathered}
$$

The convergence can be assessed at the $k^{\text {th }}$ iteration according to two criteria based on the normalized cost function value and on the stationarity of the optimal parameters:

$$
\begin{gathered}
\overline{\mathcal{J}}\left(p^{(k)}\right) \leqslant \epsilon_{1} \\
\left|p^{(k)}-p^{(k-1)}\right| \leqslant \epsilon_{2}\left|p^{(k-1)}\right|
\end{gathered}
$$

Appropriate values for the thresholds $\epsilon_{1}$ and $\epsilon_{2}$ are between $10^{-6}$ and $10^{-4}$. They must be defined jointly and consistently, depending on $(i)$ the a priori assumed accuracy of the model to update and (ii) the noise level in measurements (if known). Once one of the two previous criteria is met, the algorithm is stopped assuming its convergence (see ALG. 1).

In terms of CPU time, the repetitive calls to the solution of system (29) (whose definition depends on $\omega$ and $p$ ) must be managed at best to restrain the computational resources required for running the model updating algorithm. The computation of the CRE per subdomain, frequency and window can also be prohibitive. Hopefully, vectorized computation of CRE, convenient storage of submatrices, parallelized loops on segments $j \in \llbracket 1 ; J \rrbracket$ and frequencies $\omega \in D_{\omega}$, reduced basis for the $A x=b$ system and analytical gradient formulation strongly limit the computational resources for the computation of mCRE, allowing the full running of ALG. 1 in a couple of minutes on a personal laptop for the applications presented in the next section. 


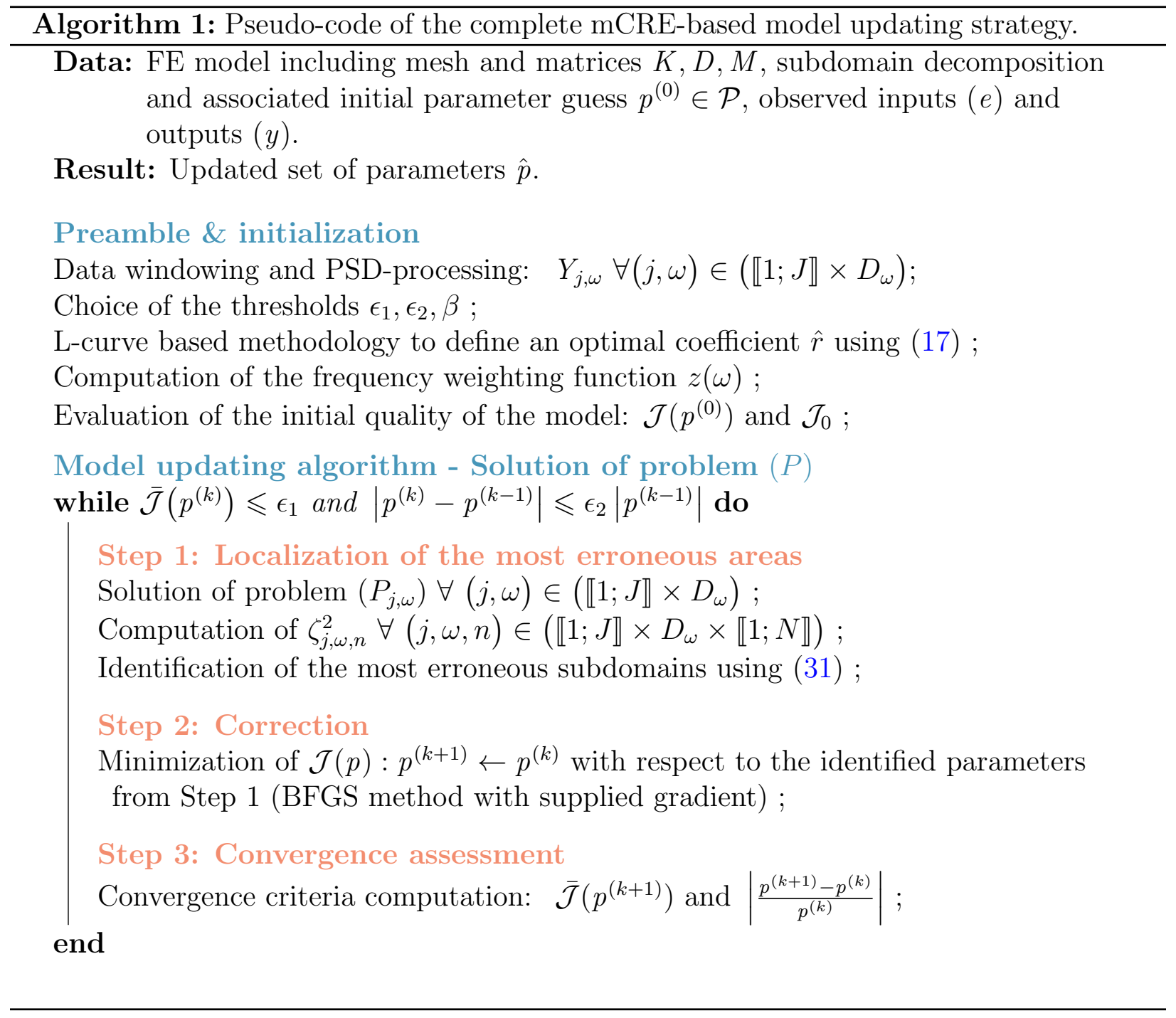

\section{Numerical applications}

In this section, numerical results are presented and discussed to assess the proposed model updating strategy. An academic plane frame model is first updated based on lowSNR measurements obtained under random ground motion. This application enables to validate the whole procedure and the robustness of the methodology with respect to known measurement noise level. Afterwards, the SMART2013 shaking-table test campaign database is used for correcting the associated FE model borrowed from [3]. The quality of the proposed corrections will be assessed by comparing the updated numerical eigenfrequencies with datadriven subspace-based experimental identification results [5].

\subsection{Application \#1: Academic plane frame subjected to random ground acceleration loading} 4.1.1. Description of the problem

We consider here the plane frame structure of FIG. 3 whose stiffness distribution is assumed unknown. This structure is clamped to a rigid moving support (shaking-table). 


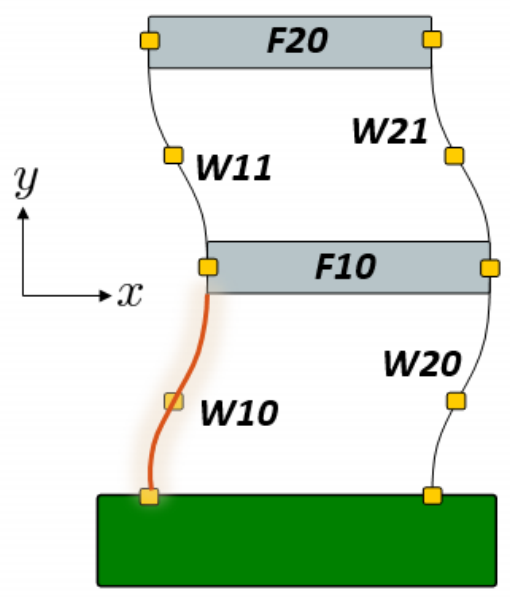

Figure 3: Plane frame model with sensors locations (yellow dots) and subdomains naming. The damaged area W10 is highlighted in orange.

The stiffness reference field (the one to be identified) presents a defect in the wall W10 whereas the initial guess is uniform (see TAB. 2). The objective of this academic example is to recover the stiffness distribution based on simulated measurements with noise of known level coming from discrete sensors scattered over the structure (yellow dots in FIG. 3).

\begin{tabular}{lcc||c}
\hline & $\begin{array}{c}\text { Healthy } \\
\text { reference }\end{array}$ & $\begin{array}{c}\text { Damaged modulus } \\
\text { in W10 }\end{array}$ & $\begin{array}{c}\text { Initial } \\
\text { guess }\end{array}$ \\
\hline Young's moduli [GPa] & 33 & 20 & 30 \\
\hline Associated parameters $[-]$ & 1.1 & 0.67 & 1.0 \\
\hline
\end{tabular}

TABLE 2: Material properties of the frame (actual configuration and initial guess).

The FE model is made of 192 elastic beam elements and built with the CEA FE simulation software CAST3M ${ }^{\circledR}[68]$. Relative time acceleration measurements in both $x$ and $y$ directions are simulated using Fast Fourier Transforms and the frequency transfer matrix of the direct dynamic problem formulated in terms of relative displacement $X$ as follows:

$$
M \ddot{X}+D \dot{X}+K X=-M \Xi \ddot{U}_{d}, \quad X=U-U_{d}
$$

where $\Xi$ is a matrix addressing the bi-axial acceleration ground motion to the associated dofs and $\ddot{U}_{d}$ the random ground acceleration input constructed as a $2 \mathrm{D}$ zero-mean white Gaussian process. As the first four modes of the structure are below $20 \mathrm{~Hz}$, a frequency bandwidth $D_{\omega}=[1 \mathrm{~Hz} ; 20 \mathrm{~Hz}]$ with $\Delta f=0.5 \mathrm{~Hz}$ has been chosen for the computation of all forthcoming results in Section 4.1. In terms of CPU time, this choice of frequency sampling and the call to a reduced basis made of the first 20 eigenmodes of the frame allowed to run the whole model updating algorithm in less than one minute on a personal laptop.

In order to assess the robustness of the methodology with respect to measurement noise, a white noise of known standard deviation is added to obtain noisy synthetic measurements. As the definition of noise level may be unclear when considering relative measurements, and 
to avoid any ambiguity in the following sections, noisy synthetic acceleration data is obtained as follows:

$$
\ddot{Y}_{\text {noisy }}(t)=\ddot{Y}(t)+\delta \operatorname{std}\left(\ddot{U}_{d}(t)\right) \eta
$$

where $\delta \in[0 ; 1]$ is the noise level, $\ddot{U}_{d}$ the input ground acceleration and $\eta$ a random vector distributed normally with zero mean and unitary standard deviation. Therefore, the added measurement noise standard deviation is equal to $\delta \operatorname{std}\left(\ddot{U}_{d}\right)$.

An intuitive decomposition of the frame is chosen: 6 subdomains are defined $\{\mathrm{W} 10, \mathrm{~W} 11$, W20, W21, F10, F20\}, one per wall and per slab (see Fig. 3). The updated stiffness model (30) is then made of $N=6$ parameters, all initialized at 1 according to the chosen initial guess (see TAB. 2).

\subsubsection{Reference model updating results}

When dealing with ideal unnoisy measurements, the model updating procedure converges quickly to the expected (and assumed unknown) parameters as shown in FIG. 4-5, illustrating the efficiency of the methodology to identify stiffness parameters from sparse measurements and to improve the correlation with reference FRFs (see FIG. 5). Note that the results of current section 4.1.2 are obtained using a default $r=0.5$ value and a single unit rectangular window (meaning no data windowing is performed).
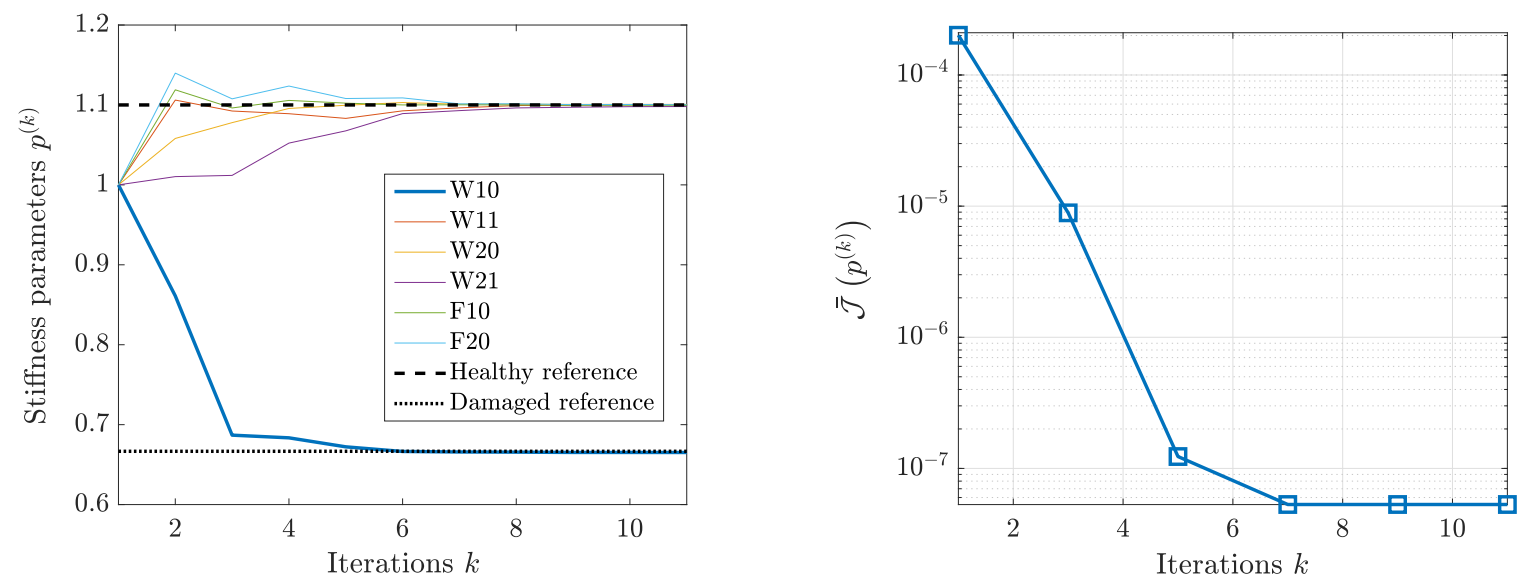

FIGURE 4: Evolution of the stiffness parameters and associated mCRE-based functional in the model updating algorithm.

\subsubsection{Enhanced robustness with respect to measurement noise}

As soon as the noise level $\delta$ increases, the classical mCRE-based model updating procedure without windowing may provide irrelevant parameter estimates, particularly due to the fact that relative measurements are naturally of low amplitude (which implies low-SNR data to process).

The results collected in TAB. 3 illustrate the effect of noise on updated parameters. The model updating procedure with a default $r=0.5$ value does not provide relevant corrections 


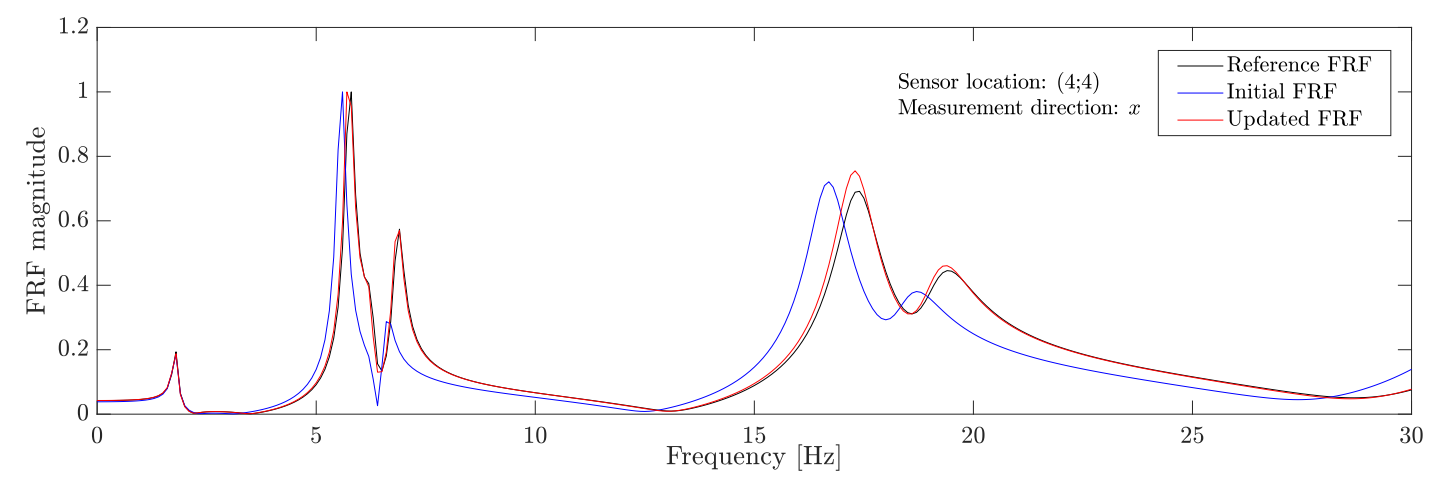

Figure 5: Experimental, initial and updated frequency response function (FRF) from a sensor located at the top of the frame.

with respect to the reference parameters. However, the model updating algorithm with an optimal $\hat{r}$ value provides a parameter estimate closer to the reference one, showing the relevance of the L-curve based metholodology proposed in 2.4.1. Fig. 6 in turn illustrates how the optimal $\hat{r}$ value (found at the intersection of the model error and measurement error curves) automatically decreases when the noise level $\delta$ grows.

TABLE 3: Comparison of parameter estimation for low-SNR measurements $(\delta=20 \%)$.

\begin{tabular}{cccc}
\hline \multirow{2}{*}{ Subdomains } & Expected & \multicolumn{2}{c}{ Updated parameters } \\
& parameters & $r=0.5$ & $\hat{r}=0.12$ \\
\hline W10 & 0.67 & 0.3550 & 0.5832 \\
W11 & 1.1 & 0.5832 & 0.9654 \\
W20 & 1.1 & 0.9683 & 1.0630 \\
W21 & 1.1 & 0.4586 & 0.9347 \\
F10 & 1.1 & 1.3619 & 1.1536 \\
F20 & 1.1 & 1.5656 & 1.1901 \\
\hline
\end{tabular}

Secondly, the use of an $H$-CMIF based frequency weighting function permits to favor automatically the eigenfrequencies of sollicitated modes. It is of crucial importance when the noise level becomes important: this particularly helps to prevent spurious increases in the mCRE frequency content between $0 \mathrm{~Hz}$ and $5 \mathrm{~Hz}$ (see Fig. 7).

Now that the algorithm enhanced capabilities for updating parameters from low-SNR measurements have been illustrated, one must finally highlight that there may be a strong dependency of the solution into the measurement noise from a statistical point of view. This makes it worthwhile to extend the convergence of model predictions towards the measurements in terms of PSDs. Indeed, when running multiple times the model updating algorithm with different measurement noise realizations, one can observe an enhanced stability in the parameter estimate when data is windowed. In FIG. 8, the width of each plotted interval represents the maximal variability of updated parameters with respect to measurement noise. It is thus clear that the data windowing preprocessing step enable the algorithm to provide more stable estimates: increasing the amount of windows $J$ in the data preprocessing step is beneficial for increasing statistical stability. However, one shall note that the number of 

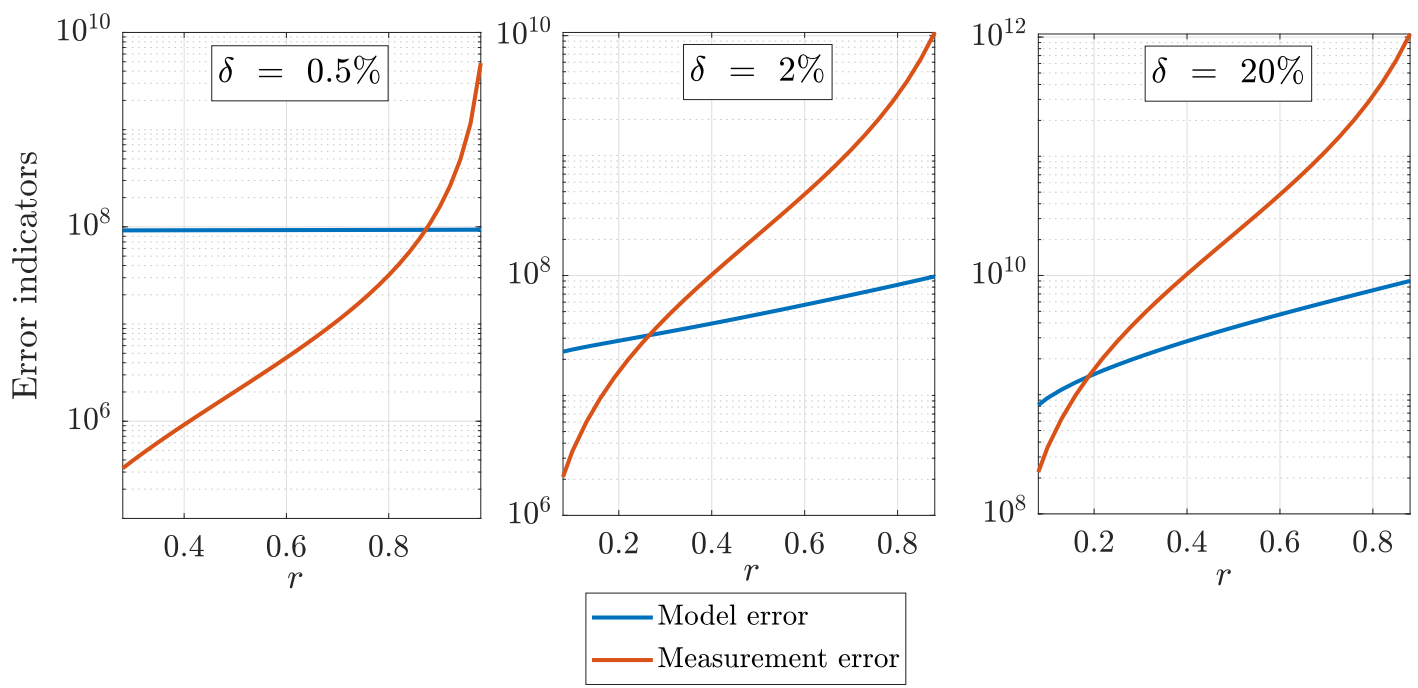

FiguRE 6: Optimal choice of $r$ according to the L-curve based methodology for balancing model and measurement errors depending on the noise level $\delta$.

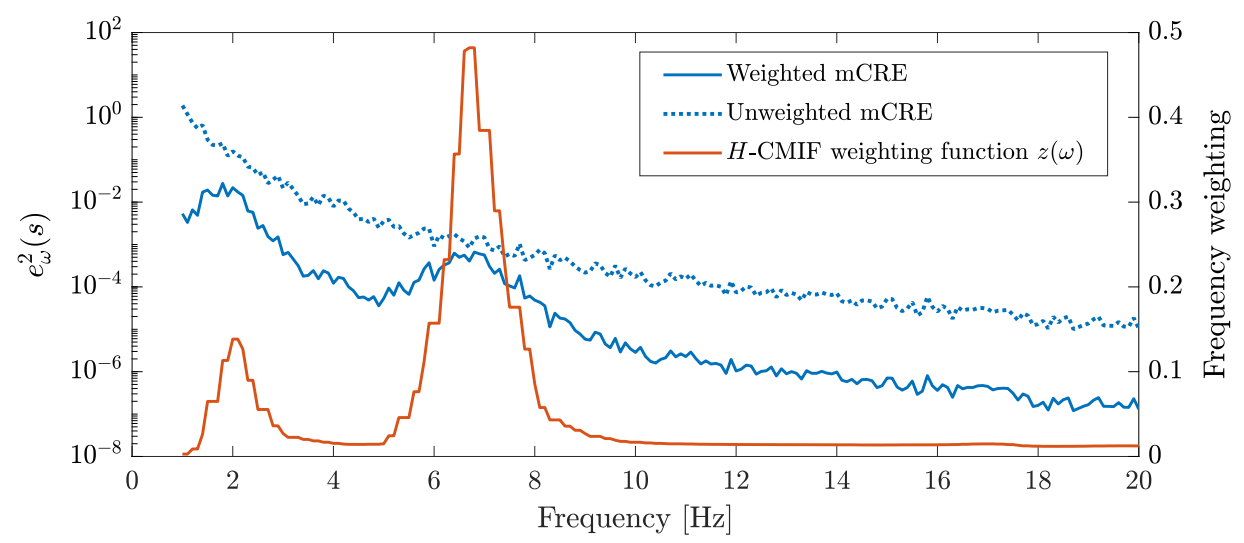

FiguRE 7: Natural emphasis of the mCRE frequency content on the experimental eigenfrequencies of sollicitated modes to enhance the robustness of the minimization procedure with respect to measurement noise.

segments cannot be indefinitely increased: statistical independence of data blocks must be guaranteed for the averaging to be meaningful [59] and CPU time constraints may occur due to the call to mathematical expectations. Noticing that reducing the time-length of windows does not drastically provide much more accurate results, a compromise must then be found between the data windowing choice and CPU time. In this work, 60\%-overlapping Blackman windows of time length up to $2 \mathrm{~s}$ have been used and always provided satisfactory results. Finally, the comparison of the intervals between subdomains (for a given data windowing configuration) shows the difference in sensitivity of the parameters to the mCRE functional: one can therefore remark that the top walls W11 and W21 are less sensitive due to the fact they do not store much mechanical energy compared to the other subdomains. 


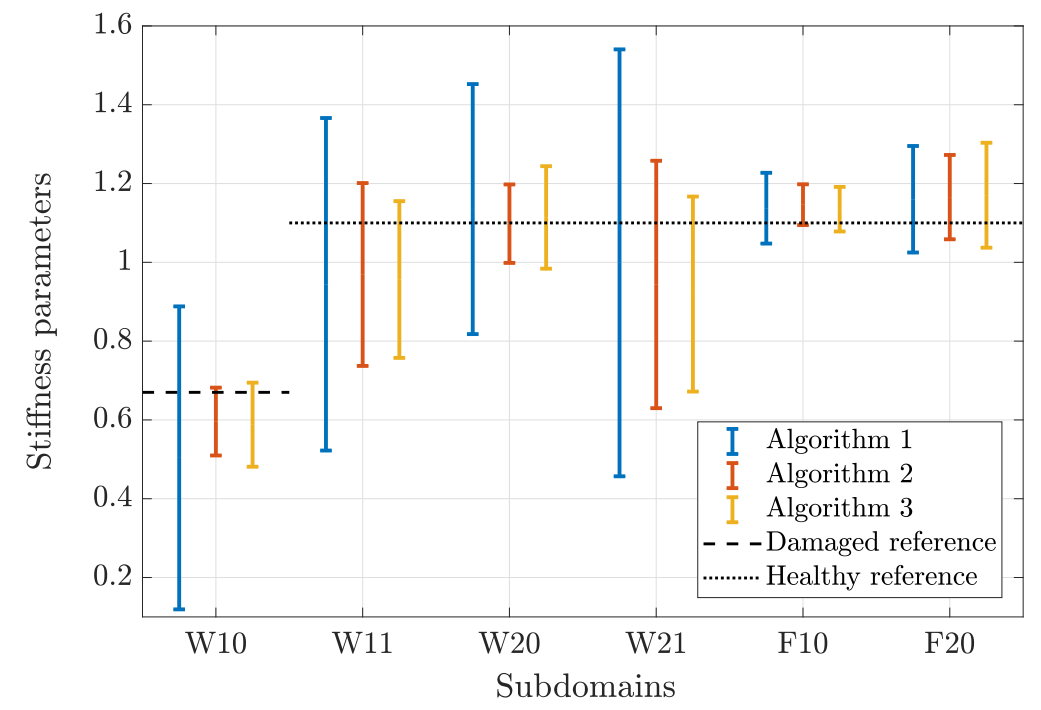

Figure 8: Statistical variability of parameter estimates with respect to measurement noise. Based on 50 different 60 s-random ground motion inputs and a $\delta=10 \%$ noise level, synthetic acceleration measurements are processed by 3 mCRE-based model updating algorithms having different data windowing: Alg. 1) Single unit rectangular window Alg. 2) Blackman-10 s data windowing, Alg. 3) Blackman-5 s data windowing.

\subsection{Application \#2: correcting the SMART2013 RC model from acceleration time histories}

\subsubsection{The SMART2013 benchmark - FE model and processed measurements}

In order to assess the vulnerability of RC structures subjected to torsional effects during seismic ground motions, the SMART2013 experimental campaign was conducted in the CEA/TAMARIS facility where a three-story $1 / 4$ reduced-scale trapezoidal RC specimen clamped on the six dofs AzALEE shaking-table has been subjected to a sequence of seismic tests. Equipped with eight $1000 \mathrm{kN}$ maximum capacity hydraulic MTS actuators, the AzALEE shaking-table can reproduce complex seismic loadings on large scale specimens. In terms of mass, nearly 34 tons of additional masses were added to the 12 tons of the structure to account for realistic floor loading and respect Cauchy-Froude's similitude law (see FIG. $9)$.

The specimen is instrumented with more than 200 sensors including 64 capacitive accelerometers of $\pm 10 \mathrm{~g}$ range scattered over the RC specimen. 48 out of the 64 accelerometers (pointed on FIG. 9 by orange circles) have been used as experimental reference for correcting the FE model. Measurements are acquired at a sample frequency of $1000 \mathrm{~Hz}$ and filtered with $400 \mathrm{~Hz}$ cut-off frequency anti-aliasing filters. A typical $\pm 0.003 \mathrm{~g}$ white noise level was observed on the accelerometers. More precisely, accelerations are recorded on corners of the trapezoid on each story (including soleplate level), while vertical accelerations are measured in-between the masses at floor levels. The displacements and accelerations of the eight hydraulic rods of the AzALEE shaking-table are also measured, providing complete and redundant access to the input imposed on the specimen.

A brief recap of the SMART2013 test campaign is given in TAB. 4. The test sequence 
consists in an alternation of bi-axial gradually damaging seismic inputs of increasing level and of random ground motions with low acceleration level chosen such that the first eigenmodes of the experimental system are excited but without adding further damage to the RC specimen. Complementary information can be found in [3-5, 69].
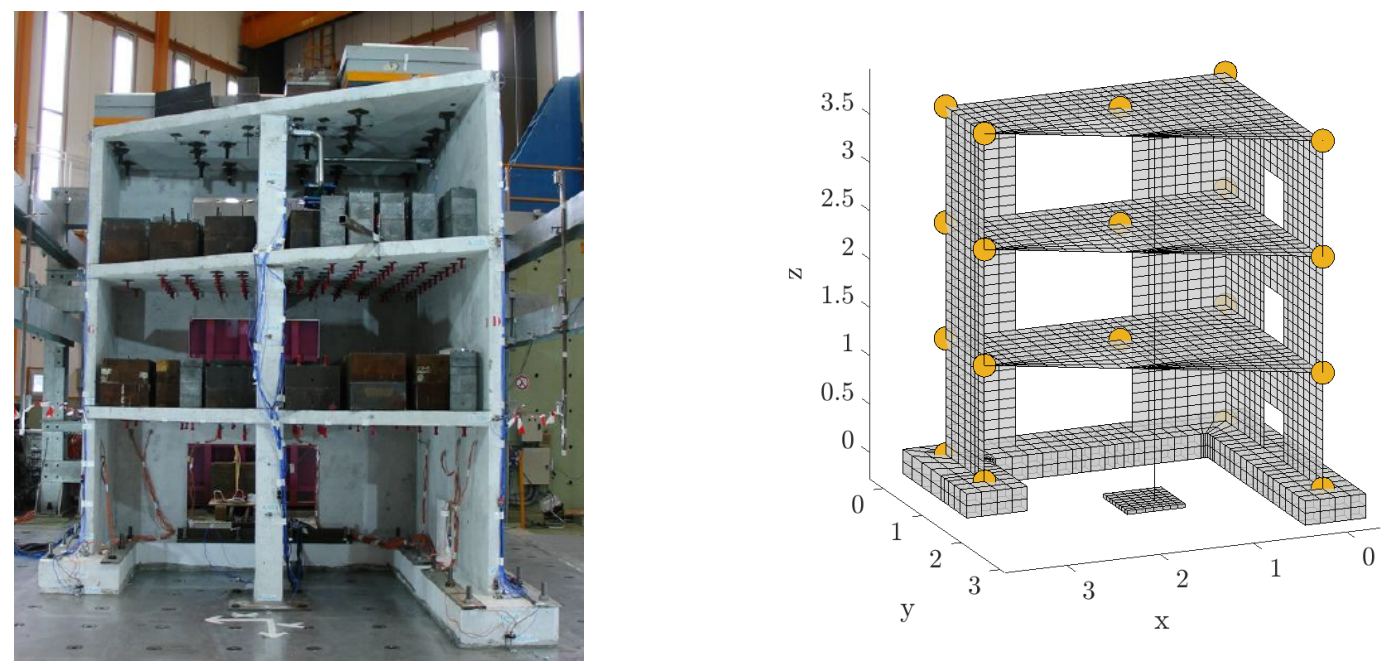

Figure 9: The SMART RC specimen anchored to the AzALEE shaking-table and the associated FE model developed in $\mathrm{CAST}_{3} \mathrm{M}^{\complement}$. The orange circles on the mesh indicate the location of tri-axial accelerometers used in the model updating strategy.

The FE model considered herein has been developed in the CAST3M ${ }^{\circledR}$ [68] FE framework using multi-layered shell and Timoshenko multi-fiber beam elements (5362 nodes, 24202 elements, 20661 degrees of freedom - see Fig. 9) and fully decribed in [3, 4]. Note that, following the conclusions drawn by [5], the AzALEE shaking-table is not included in the model; the structure will be assumed to be cantilevered to a moving rigid support.

In spite of the huge numerical efforts made for modeling the experimental SMART2013 test-results, the recorded data itself has never been used for model updating purposes. Let us however mention the preliminary work of [70] that assessed the potential of CRE on simulated measurements to identify damage deliberately introduced into a simplified model of the RC specimen. Anyway, the call to a model updating procedure makes sense when observing the strong gaps between the predicted and the experimental eigenfrequencies (see TAB. 5 and [3]).

\subsubsection{Updating of the initial configuration - first results and comparison with classical fre- quency least-square minimization}

Knowing the large gap in terms of natural frequencies between model predictions and available measurements (even at the beginning of the campaign, see TAB. 5), a single global stiffness parameter is updated herein. Due to the fact that the seismic loading is bi-axial, only the sensors measuring accelerations along $x$ and $y$ directions are considered in the model updating strategy. The analysis of acceleration time series and associated single-window FFT (see FIG. 10 and TAB. 4) confirms the low-frequency dynamics of the measurements and therefore justifies the use of the extended mCRE-based model updating algorithm previoulsy 


\begin{tabular}{|c|c|c|}
\hline \multicolumn{2}{|c|}{ Phase 1: SMART2008 inputs - PGA $(x, y)=(0.2 g, 0.2 g)$} & \multirow{2}{*}{$\frac{\delta[\%}{22.6}$} \\
\hline Run \#6 & Broad-band bi-axial signal $(x+y) 0.02 g$ RMS & \\
\hline Run \#7 & Seismic signal - 50\% & - \\
\hline Run \#8 & Broad-band bi-axial signal $(x+y) 0.02 g$ RMS & 29.0 \\
\hline Run \#9 & Seismic signal - $100 \%$ & - \\
\hline \multicolumn{3}{|c|}{ Phase 2: Northridge main shock signal - PGA $(x, y)=(1.78 g, 0.99 g)$} \\
\hline Run \#10 & Broad-band bi-axial signal $(x+y) 0.02 g$ RMS & 23.8 \\
\hline Run \#11 & Seismic signal - $11 \%$ & - \\
\hline Run \#12 & Broad-band bi-axial signal $(x+y) 0.02 g$ RMS & 31.1 \\
\hline Run \#13 & Seismic signal - $22 \%$ & 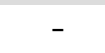 \\
\hline Run \#14 & Broad-band bi-axial signal $(x+y) 0.02 g$ RMS & 26.1 \\
\hline Run \#15 & Seismic signal - 22\% & - \\
\hline Run \#16 & Broad-band bi-axial signal $(x+y) 0.02 g$ RMS & 28.5 \\
\hline Run \#17 & Seismic signal - 44\% & - \\
\hline Run \#18 & Broad-band bi-axial signal $(x+y) 0.02 g$ RMS & 28.0 \\
\hline Run \#19 & Seismic signal - $100 \%$ & - \\
\hline \multicolumn{3}{|c|}{ Phase 3: Northridge after-shock signal - PGA $(x, y)=(0.37 g, 0.31 g)$} \\
\hline Run \#20 & Broad-band bi-axial signal $(x+y) 0.02 g$ RMS & 20.1 \\
\hline Run \#21 & Seismic signal $33 \%$ & - \\
\hline Run \#22 & Broad-band bi-axial signal $(x+y) 0.02 g$ RMS & 26.0 \\
\hline Run \#23 & Seismic signal $100 \%$ & - \\
\hline Run \#24 & Broad-band bi-axial signal $(x+y) 0.02 g$ RMS & 22.5 \\
\hline
\end{tabular}

TABLE 4: Synthesis of the SMART2013 shaking-table test campaign and computation of noise level $\delta$ for the bi-axial random tests.

described. Practically, the analysis of the frequency content of Fig. 10 enables to choose $D_{\omega}=[1 \mathrm{~Hz} ; 30 \mathrm{~Hz}]$ with $\Delta f=0.1 \mathrm{~Hz}$. Note that the signal-to-noise ratio (SNR) for lowPGA inputs of the SMART2013 database is so important (although intrinsic sensors noise level is low) that a uniform frequency weighting function and a $r=0.5$ default value lead to non-physical or divergent results. The variations of the functional $\overline{\mathcal{J}}$ with respect to $p$ and $r$ is plotted in FIG. 11 to illustrate once again the non-negligible impact of $r$ on the parameter estimate provided by the updating algorithm; the L-curve based methodology explained in Section 2.4.1 is a necessary preliminary step to handle at best the whole SMART2013 recorded database considered in the model updating strategy. The addition of a normalized $H$-CMIF frequency weighting function, as detailed in Section 2.4.2, enables to exploit at best all the available information from measurements minimizing spurious noise effects.

One could legitimately wonder about the performance of the mCRE-based proposed methodology when compared to classical Frequency Least-Square (FLS) minimization written in the frequency domain: $\overline{\mathcal{J}}_{F L S}(p)=\int_{D_{\omega}}\left\|\Pi U_{\omega}(p)-Y_{\omega}\right\|^{2} \mathrm{~d} \omega$. Note that it is shown mathematically in [40] that one can see the FLS minimization like a particular limit case of the mCRE-based model updating algorithm with $r \rightarrow 1$. The plot of both mCREand FLS-based functionals in FIG. 12 illustrates the enhanced convexity properties of the mCRE-based cost function. Therefore, it yields an easier convergence towards the optimal 


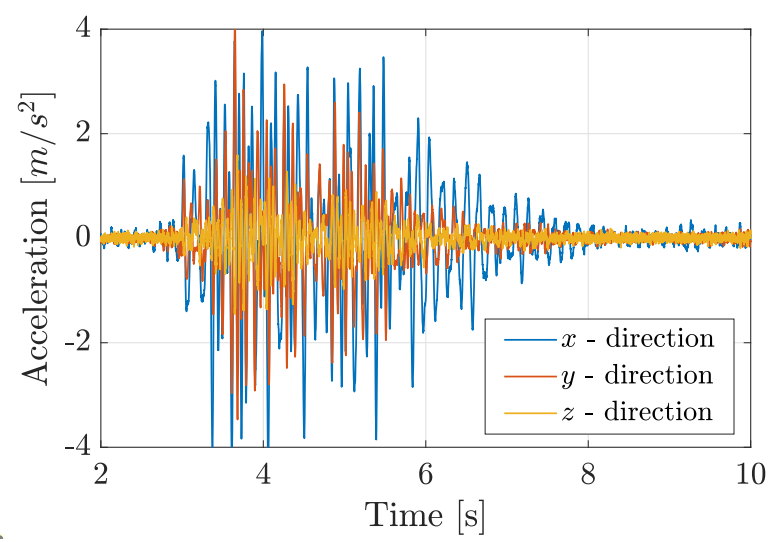

(a) Relative acceleration time series between the top of the structure and the AzALEE shaking-table.

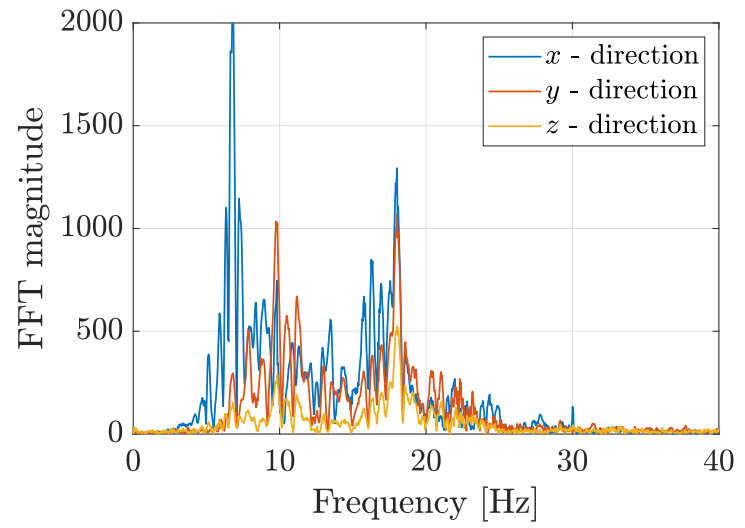

(b) Relative acceleration single window FFT between the top of the structure and the soleplate.

Figure 10: SMART2013 - Run \#7: Relative measurements between the soleplate and a $3^{\text {rd }}$ story sensor.

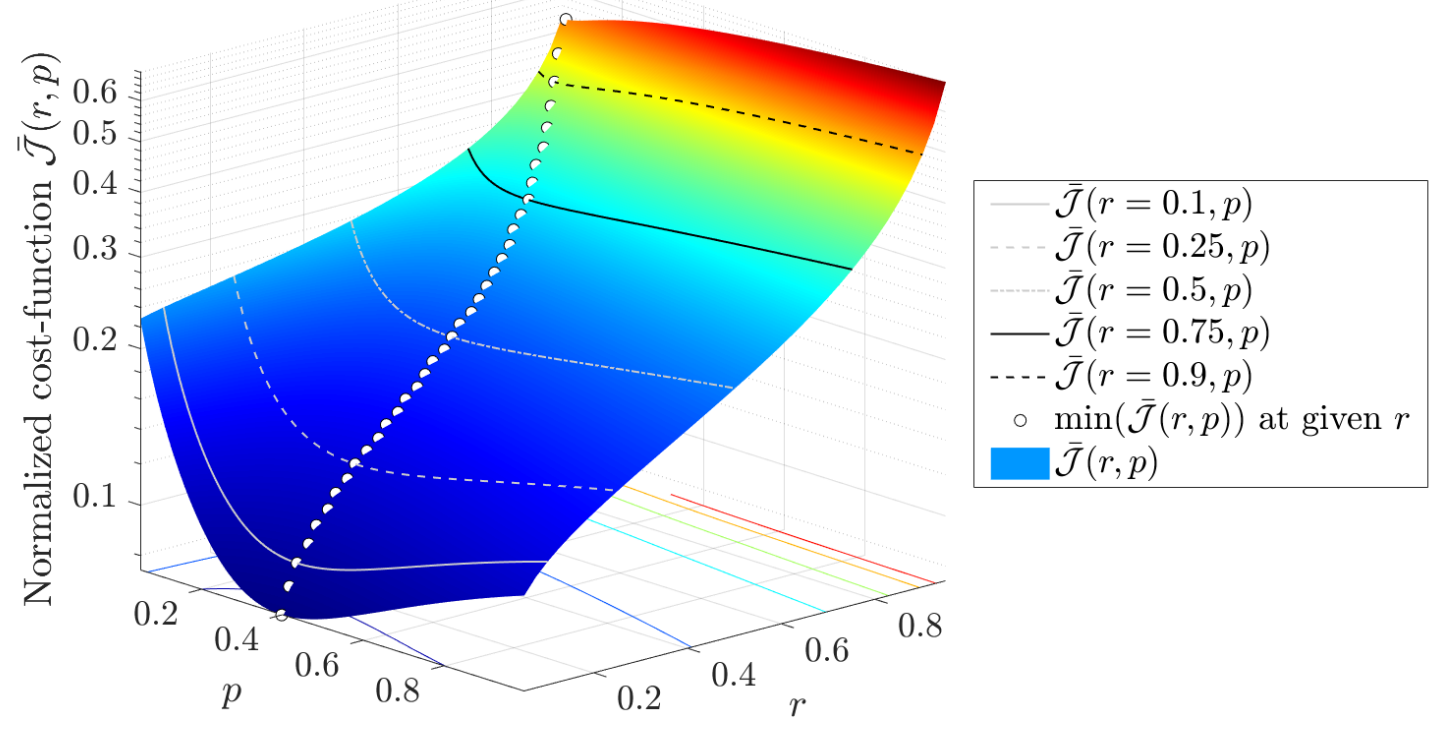

Figure 11: SMART2013 - Run \#7 - Influence of the coefficient $r$ on the model updating procedure. The convexity of the mCRE functional is always guaranteed but the minimum value is significantly modified with $r$ (the white circles indicate the minimum of the cost function for a given $r$ ).

set of parameters. Besides, the computation of the updated eigenfrequencies based on the corrected FE model in TAB. 5 shows that the mCRE-based algorithm provides much more reliable results (in better match with experimental eigenfrequencies).

An additional quantitative appreciation of the model updating results on the whole frequency range can be obtained by comparing the experimental reference to models in terms of $H$-CMIF. Several normalized $H$-CMIFs are displayed in FIG. 13:

(i) Reference experimental $H$-CMIF computed from accelerometers (see (18-20)),

(ii) $H$-CMIF from the initial model $(p=1)$,

(iii) $H$-CMIF from the mCRE-based updated model with default $r=0.5$, 


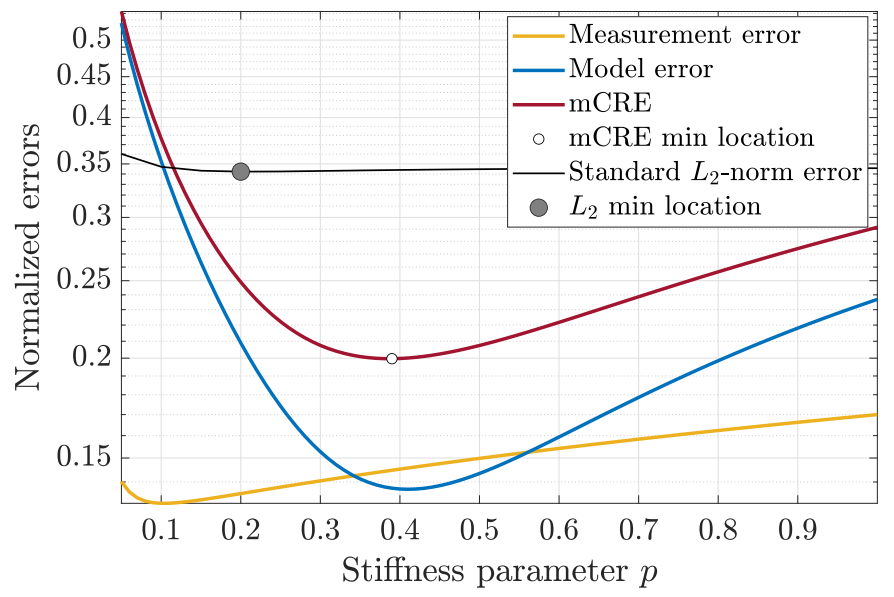

FiguRE 12: SMART2013 - Run \#7 - Identification of a global stiffness parameter using the mCRE-based model updating algorithm. An enhanced convexity shows the relevance of the methodology compared to a frequency total least-square error ( $L_{2}$-norm between model and measurements).

\begin{tabular}{c|c|cc|cc|cc}
\hline \multirow{2}{*}{$\begin{array}{c}\text { Mode } \\
\text { number }\end{array}$} & Experimental & \multicolumn{2}{|c|}{ Initial } & \multicolumn{2}{c|}{ mCRE updated } & \multicolumn{2}{c}{ FLS updated } \\
\cline { 3 - 8 } & frequency & Frequency & Error & Frequency & Error & Frequency & Error \\
\hline \hline 1 & 6.28 & 9.10 & 44.8 & 5.68 & 14.9 & 4.07 & 39.1 \\
2 & 9.22 & 15.72 & 70.5 & 9.82 & 6.5 & 7.03 & 23.7 \\
3 & 17.6 & 31.77 & 80.5 & 19.84 & 12.7 & 14.21 & 19.3 \\
\hline
\end{tabular}

TABlE 5: Model updating results obtained for SMART2013 run \#7 - Comparison of the first eigenfrequencies (in [Hz]) and relative errors (in [\%]) between experimental reference [5], initial model and updated models from the mCRE-based and frequency least-square (FLS) model updating algorithms.

(iv) $H$-CMIF from the mCRE-based updated model with optimal $\hat{r}$,

(v) $H$-CMIF from the FLS-updated model.

The numerical $H$-CMIFs (ii-v) above correspond to the dominant singular value of the transfer function $H$ computed using the FE matrices $M, D, K$. From these plots one can conclude that the parameter estimates provided by the mCRE-based algorithms are more physically relevant with respect to the expected frequency content; the corrected model $H$-CMIFs get closer to the experimental reference, especially in the vicinity the two first eigenfrequencies. The choice of a single parameter model is obviously too simplistic to perfectly recover the experimental frequency content. A better adequacy with higher eigenfrequencies could certainly be obtained by updating several parameters simultaneously, with risks of having convergence issues. The choice of an optimal $\hat{r}$ permits an improved estimation of the two first eigenfrequencies, whose eigenmodes are essential in the low-frequency specimen dynamic response.

Remark: Note that no data windowing has been performed previously considering that run \#7 time series cannot be assumed ergodic and stationary. The enhanced statistical stability of data windowing will be observed in the next section when considering low-SNR random measurements from even runs of the test-campaign. 


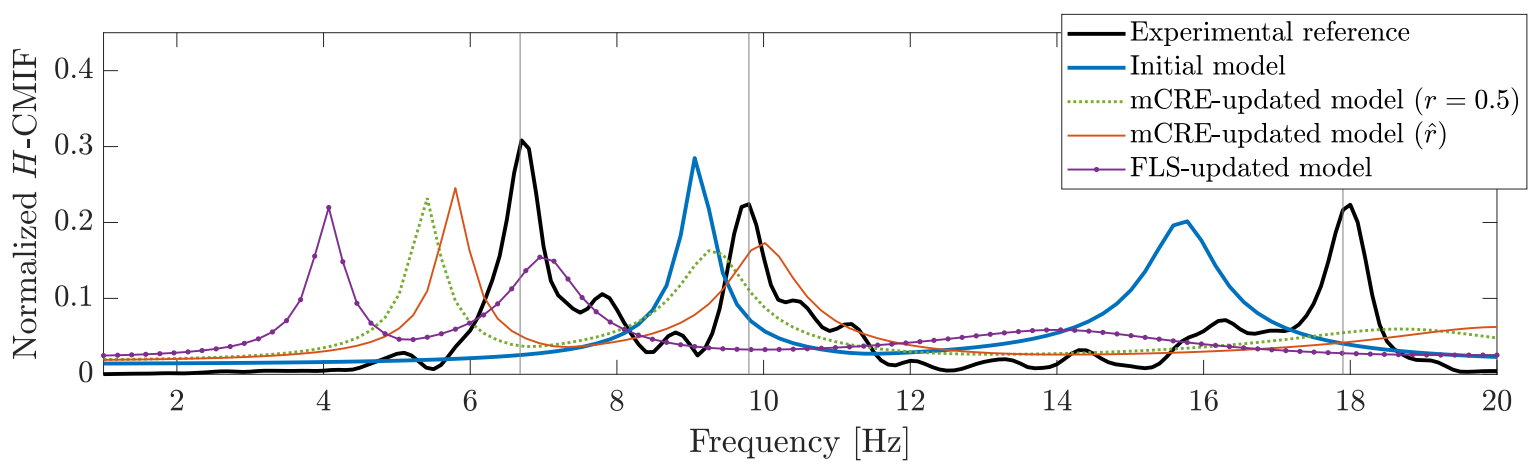

Figure 13: SMART2013 - Run \#7: H-CMIF comparison between experimental reference and model predictions before/after updating. The mCRE-based model updating algorithms provide more accurate results than FLS minimization in terms of $H$-CMIF: a compromise is made for fitting at best the experimental frequency peaks (marked with grey vertical lines).

\subsubsection{Offline correction of the FE model towards the entire SMART2013 test-campaign}

In what follows, the mCRE-based model updating procedure will be sequentially used for correcting the FE model along the whole SMART2013 test campaign. In practice:

- the measurements from each run are consecutively processed by the model updating algorithm, meaning that an updated parameter value is associated to each run ;

- when processing run \#n, the final result from the previous run \#(n-1) is used as initial guess in order to guarantee closeness to the 'optimal' parameter value.

Due to the fact that damaging runs time series inputs cannot be assumed as ergodic and stationary signals, the extended mCRE-based model updating algorithm with data windowing (which favors data-model correlation in terms of PSD) is exclusively assessed on the low-PGA runs. Two variants of the model updating algorithms are thus run:

(a) Model updating using a swingle unit-rectangular window for data windoing, which is convenient for all testings and do not guarantee optimal stability with respect to measurement noise.

(b) Model updating of the random low-PGA runs (even runs from TAB. 4) using 60\%overlapping Blackman-5 $\mathrm{s}$ data windowing (illustrated in FIG. 2).

FIG. 14 presents the evolution of the updated three first eigenfrequencies (squares) compared to the experimental reference from [5] (connected circles) using a single parameter stiffness model, whose estimated optimal values are also specified (right subplots). In both cases, the model updating procedure provides relevant correcting actions, confirmed by the proximity of the reference eigenfrequencies given by [5] (connected circles in Fig. 14). The progression of damage can be related to the decreasing values of the updated parameter throughout the different runs.

The study of the frequency drop highlights two stages in the test campaign where parameters remain globally constant: runs \#6 to \#12 (phase 1: SMART2008 inputs), and \#20 to \#24 (phase 3: after-shock analysis). One can remark in each of them that the enhanced 

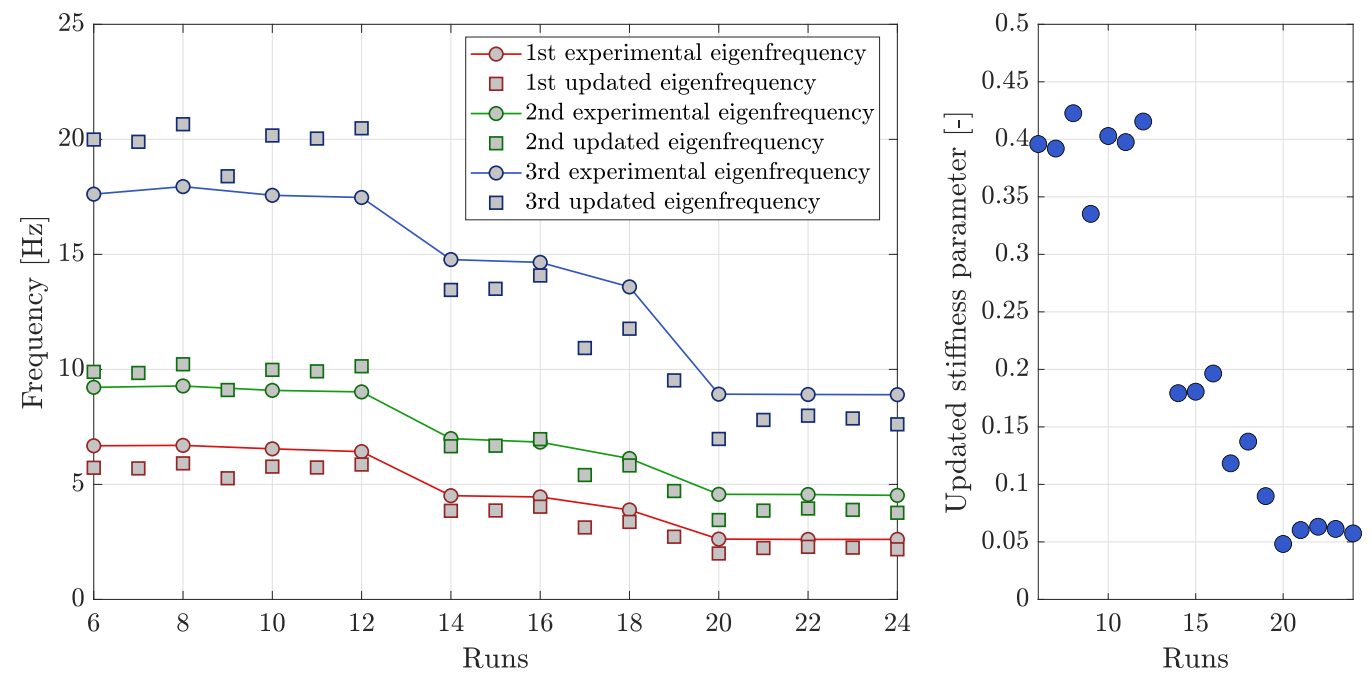

(a) mCRE-based model updating results without data windowing.
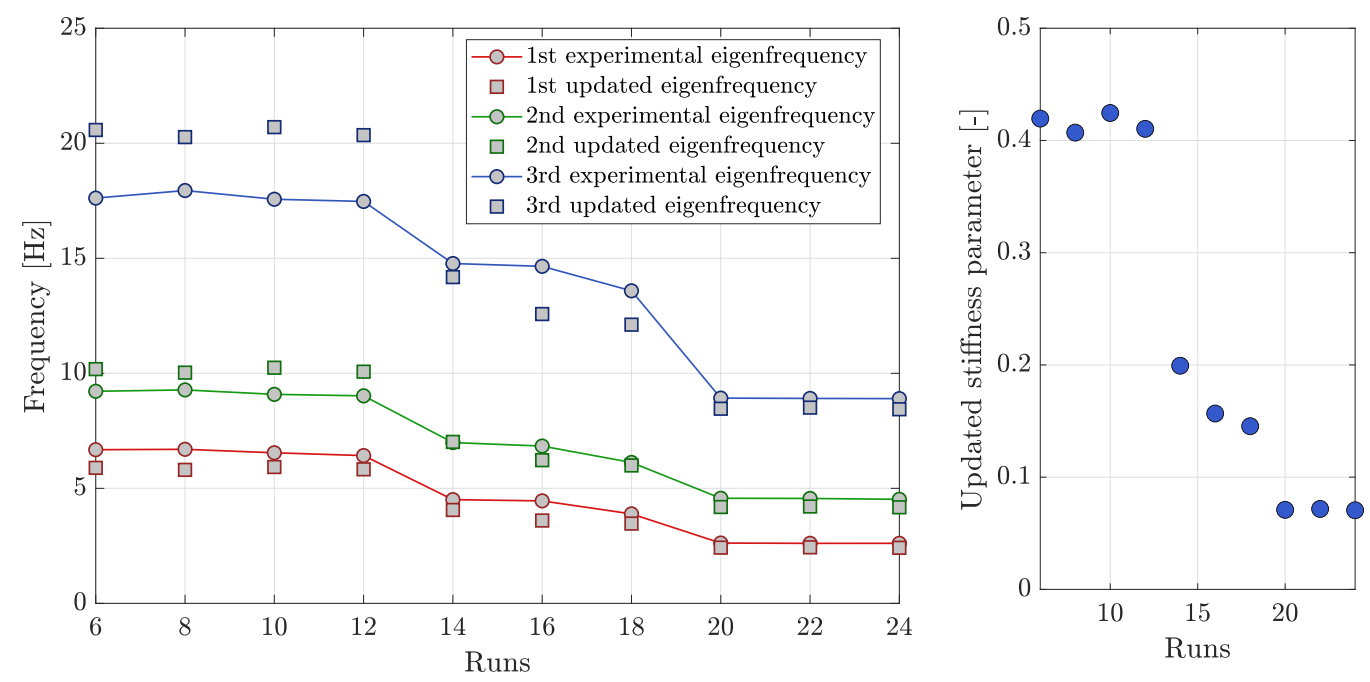

(b) mCRE-based model updating results with data windowing for the broad-band low-PGA tests exclusively. 5 s-Blackman windows have been used.

FIgURE 14: Correction of the SMART2013 FE model using mCRE-based model updating algorithms during the entire test-campaign - tracking the modal signature and parameter estimate.

statistical robustness of data windowing (comparing FIG. 14a and 14b) confers a better stability of the parameter estimate, as previously observed in the first application of this paper. Anyway, one has to notice that a correctly updated (linear) stiffness FE model is able to follow the state of a structure during the entire campaign, in which many nonlinear phenomena occur.

\subsubsection{Towards adaptive parametrization and subdomains refinement}

Until now, the updating procedure has only been focusing on one global stiffness parameter, providing relevant tracking of the first eigenfrequencies drop, which is directly related to the evolution of the overall damage state of the specimen. This global damage assessment 
is already promising for better control of shaking-table actuators. However, it is certain that the corrections of the FE stiffness matrix proposed herein may lack of relevance for local damage detection purposes as the overall weighting of the stiffness matrix does not give access to any local information. Therefore, the feasibility of the identification of several parameters naturally arises. Indeed, now that the model is globally updated, a next step would consist in defining smaller subdomains in order to evaluate the damage state more locally. It raises new problems, particularly regarding the relative accuracy of the algorithm when identifying several parameters simultaneously and its capability to update accurately all of them.

We now consider the FE model to be split in four subdomains whose stiffness FE submatrices are obtained by assembling the elementary contributions of the elements belonging to each one of them. They are displayed in FIG. 15.
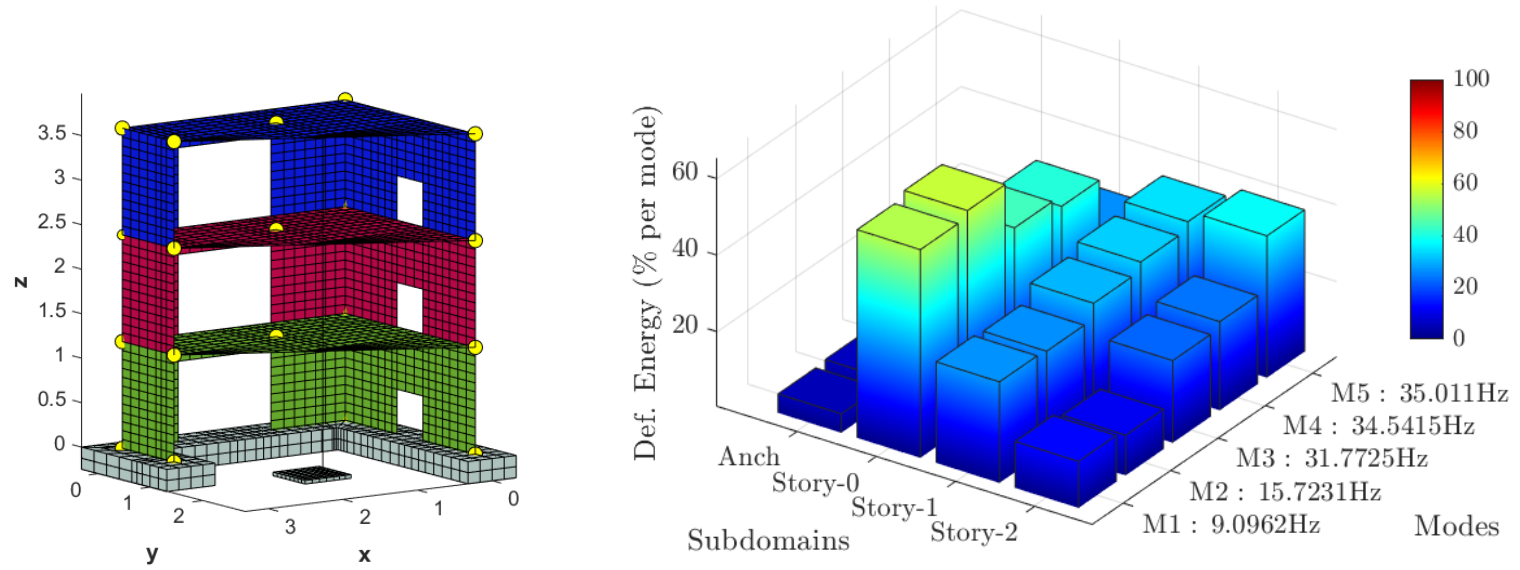

FIgURE 15: Four-subdomains decomposition of the SMART2013 specimen FE model and associated mechanical strain energy contributions per subdomain and per mode.

To the extent that the CRE distribution per subdomain and mechanical strain energy are intrinsically related, a first preliminary analysis of the modal deformation energy contributions per subdomain can be performed remarking that, if among all, certain subdomains have a very low-energy contribution compared to the others, then it will be probably difficult to properly update the associated parameters properly. For the initial FE model, the modal strain energies per subdomain are displayed in FIG. 15 for the first five eigenmodes. One can notice that the contribution of the anchorage and 3rd floor subdomains are of less importance compared to the global mechanical strain of the structure. Therefore, the lack of sensitivity of the associated parameters may lead to an uncertain updated value.

To confirm this statement, a 2D map of the mCRE functional with respect to the 1st and 2nd floor stiffness parameters has been plotted in FIG. 16 based on run \#6 data. The amount of possible couples of parameters that are relevant in the mCRE sense shows that the algorithm will hardly be able to provide a consistent minimum value. Indeed, the mCRE functional is sensitive to both parameters, but the lack of local information (i.e. the low density of sensors) limits the capability of mCRE to identify parameters accurately, even if they are selected as most sensitive. Although a large number of sensors are spread over 
the specimen, the experimental information they bring is unfortunately not rich enough to locally quantify the damage state of the specimen, even if the model updating process is restrained to the most sensitive areas.

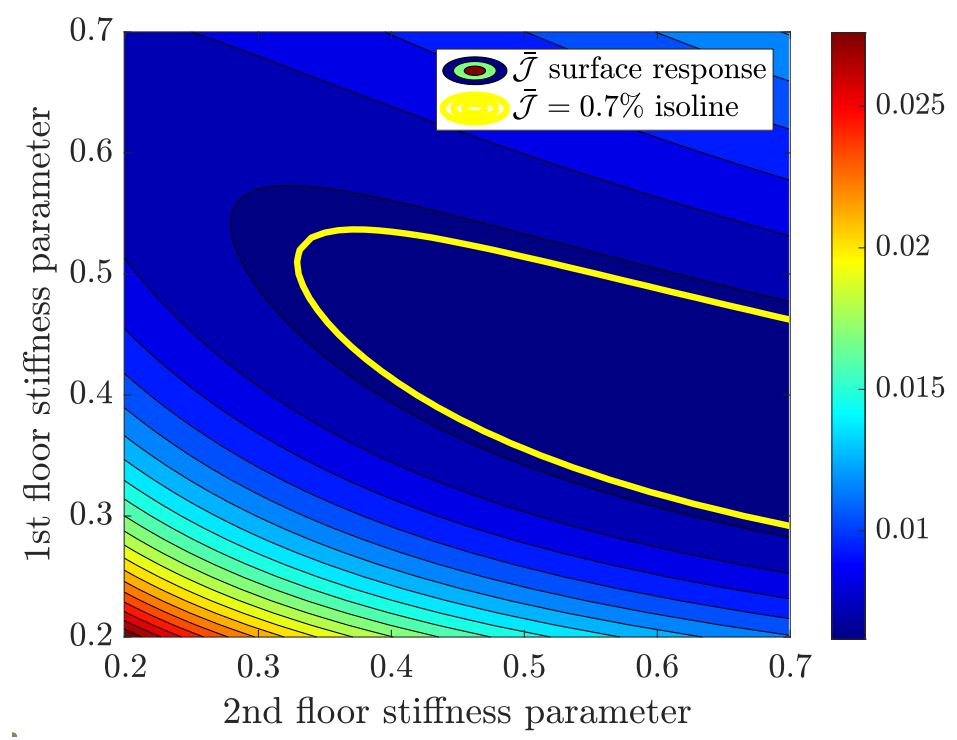

FiguRE 16: 2D map of the mCRE functional $\overline{\mathcal{J}}$ with respect to the 1st and 2nd floor stiffness parameters from Run \#6 database. An isoline at $0.7 \%$ has been emphasized to show the (numerous) relevant set of parameters in the mCRE sense.

To assess the choice of subdomains for the mCRE-based model updating, one can finally conclude that a compromise must be done between:

(i) the dimension of the parameter space $\mathcal{P}$,

(ii) the richness and sparsity of available measurements,

(iii) and the heterogeneous sensitivity of subdomains with respect to the mCRE functional,

to avoid sub-optimal parameter estimates corresponding to local mCRE minima. The definition of an adaptive model updating strategy that exploits measurements at best to define an optimal (and economous) parametrization is a research topic of interest for forthcoming studies. Very few references have studied the coupling of adaptive processes with inverse problems [71-74]. The strong asset of using mCRE in such a context is the possibility to exploit the CRE as a local refinement indicator, as done for classical verification/validation purposes [75]. 


\section{Conclusions}

In this paper, a robust energy-based model updating framework from data acquired in low-frequency dynamics was proposed. A strategy for correcting FE stiffness models by exploiting at best a set of available measurements a posteriori was discussed. The model updating methodology based on the so-called modified Constitutive Relation Error has been (i) extended to random processes with a formulation of the cost function to be minimized in terms of PSDs and (ii) tuned for an optimal integration of low-SNR measurements in the algorithm. A L-curve based methodology has been implemented to automatically define an optimal balance between the model and measurement error and the $H-\mathrm{CMIF}$ has been exploited as a frequency weighting function to naturally emphasize the experimental eigenfrequencies of the tested structure. The first application of the implemented algorithm for updating an academic plane frame model subjected to random ground motion validated the robustness of the approach with respect to known noise level. The second application was dedicated to the SMART2013 shaking-table test campaign database. Updating a single-global stiffness parameter highlighted the possibility to recover the first eigenfrequencies drops of a RC specimen submitted to a sequence of damaging seismic loadings; the eigenfrequencies of the corrected FE model are in good correlation with former data-driven subspace-based identification results.

The general model updating framework provided in this paper was applied to the earthquake engineering context but it is perfectly suited for many other applications involving vibratory loadings and low-SNR measurements: vibration-based damage detection, ageing of civil engineering structures, structural health monitoring, fatigue of mechanical components, and more generally systems submitted to vibratory phenomena potentially coupled with thermal evolution. Indeed, once reliable and unreliable quantities of the reference problem have been distinguished by engineering judgement, a mCRE-based cost-function can be built and minimized following the methodology presented in this paper. In the perspective of more accurate damage detection, a finer stiffness parametrization must be introduced. Unfortunately, the algorithm has not been able to provide relevant results with more than one subdomain in the SMART2013 case because of the sparse density of available recordings. Forthcoming research work will be dedicated to extend the proposed mCRE-based model updating algorithm to an adaptive model updating process as the CRE map can provide local information regarding the intrinsic validity of the model itself. The possibility to use the CRE as a parameter space refinement indicator will be the topic of a forthcoming paper.

Finally, the developed tools will be reinvested within a complete data-driven strategy (see FIG. 17), where coupling with data assimilation techniques and adaptive model-based control theory are projects for future works. 


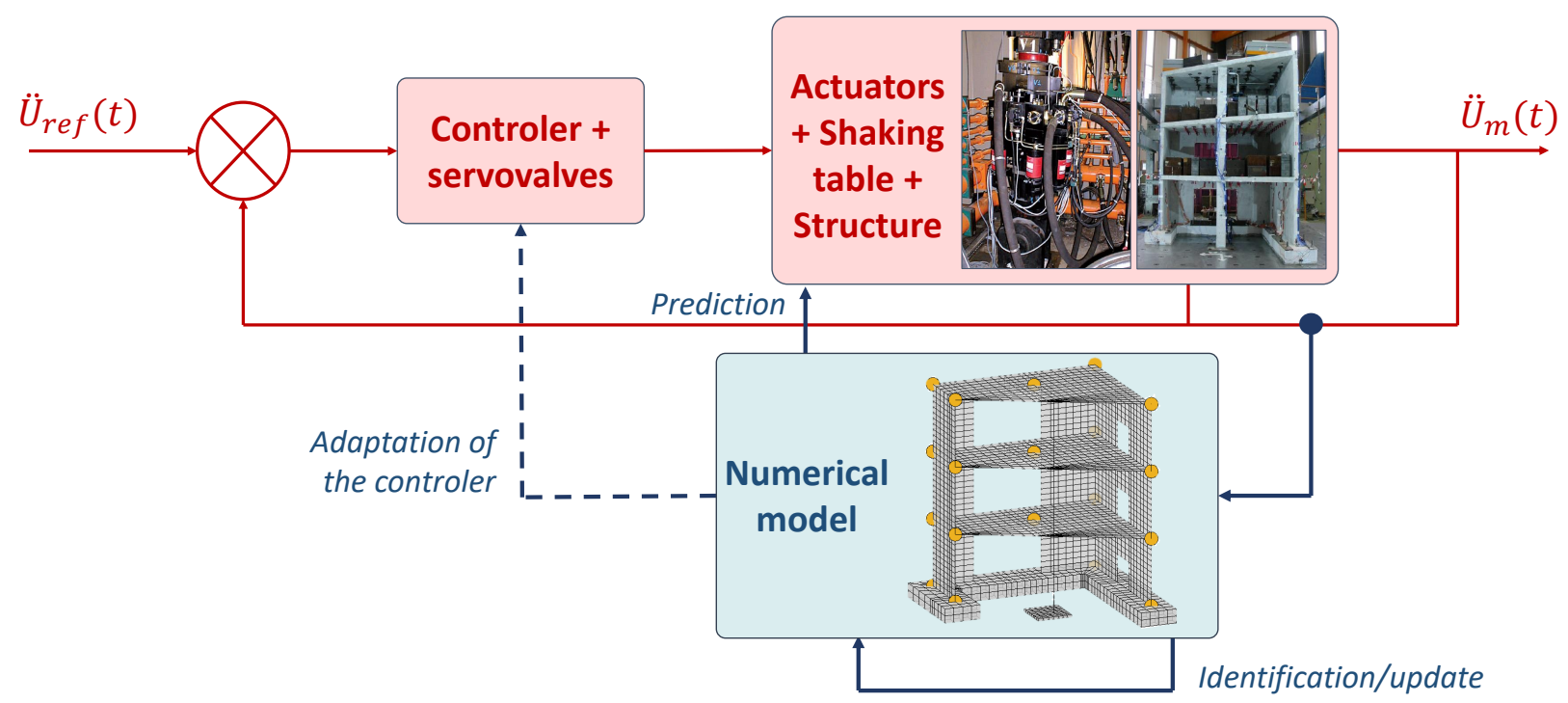

FiguRE 17: Towards the introduction of a numerical updated model in shaking-table control strategies.

\section{Acknowledgements}

EDF and CEA are deepfully acknowledged for giving access to the SMART2013 test campaign database. F. Voldoire (EDF) is more personally thanked for the fruitful comments that helped improving the quality of the paper. The C\&S reviewers are finally acknowledged for their numerous comments and advices, that were of great help to shape this contribution in its current form.

\section{References}

[1] O. Avci, O. Abdeljaber, S. Kiranyaz, M. Hussein, M. Gabbouj, D. J. Inman, A review of vibration-based damage detection in civil structures: From traditional methods to Machine Learning and Deep Learning applications, Mechanical Systems and Signal Processing 147 (2021) 107077. doi:10.1016/j .ymssp. 2020.107077.

URL https://linkinghub.elsevier.com/retrieve/pii/S0888327020304635

[2] A. Deraemaeker, E. Reynders, G. De Roeck, J. Kullaa, Vibration-based structural health monitoring using output-only measurements under changing environment, Mechanical Systems and Signal Processing 22 (1) (2008) 34-56. doi:https://doi.org/10.1016/j.ymssp. 2007. 07.004.

URL https://www.sciencedirect.com/science/article/pii/S0888327007001148

[3] B. Richard, S. Cherubini, F. Voldoire, P.-E. Charbonnel, T. Chaudat, S. Abouri, N. Bonfils, SMART2013: Experimental and numerical assessment of the dynamic behavior by shaking table tests of an asymmetrical reinforced concrete structure subjected to high intensity ground motions, Engineering Structures 109 (2016) 99-116. doi:10.1016/j.engstruct.2015.11. 029. 
[4] B. Richard, F. Voldoire, M. Fontan, J. Mazars, T. Chaudat, S. Abouri, N. Bonfils, SMART2013: Lessons learned from the international benchmark about the seismic margin assessment of nuclear rc buildings, Engineering Structures 161 (2018) 207-222. doi:https: //doi.org/10.1016/j.engstruct.2018.02.023.

[5] P.-E. Charbonnel, Fuzzy-driven strategy for fully automated modal analysis: Application to the SMART2013 shaking-table test campaign, Mechanical Systems and Signal Processing 152 (2021) 107388. doi:https://doi.org/10.1016/j.ymssp.2020.107388.

URL http://www.sciencedirect.com/science/article/pii/S0888327020307743

[6] A. Tarantola, Inverse problem theory and methods for model parameter estimation, SIAM, 2005. doi:10.1137/1.9780898717921.

URL https://doi.org/10.1137/1.9780898717921

[7] J. E. Mottershead, M. I. Friswell, Model updating in structural dynamics: A survey, Journal of Sound and Vibration 167 (1993) 347-375. doi:10.1006/jsvi.1993.1340.

[8] F. M. Hemez, S. W. Doebling, Review and assessment of model updating for non-linear, transient dynamics, Mechanical Systems and Signal Processing 15 (2001) 45-74. doi:10. 1006/mssp. 2000.1351.

[9] M. Bonnet, A. Constantinescu, Inverse problems in elasticity, Inverse Problems 21 (2005). doi:10.1088/0266-5611/21/2/R01.

[10] E. Simoen, G. D. Roeck, G. Lombaert, Dealing with uncertainty in model updating for damage assessment: A review, Mechanical Systems and Signal Processing 56 (2015) 123-149. doi: $10.1016 / j \cdot y m s s p .2014 .11 .001$.

URL http://dx.doi.org/10.1016/j.ymssp.2014.11.001

[11] A. N. Tikhonov, V. Y. Arsenin, Solution of ill posed problems, Wiley, New York (1977) 521524.

URL https://www .ams.org/journals/bull/1979-01-03/S0273-0979-1979-14602-0/

[12] V. A. Morozov, The error principle in the solution of operational equations by the regularization method, USSR Computational Mathematics and Mathematical Physics 8 (2) (1968) 63-87. doi:10.1016/0041-5553(68)90034-7.

URL https://www.sciencedirect.com/science/article/pii/0041555368900347

[13] H. Ahmadian, J. E. Mottershead, M. I. Friswell, Regularisation methods for finite element model updating, Mechanical Systems and Signal Processing 12 (1998) 47-64. doi:https: //doi.org/10.1006/mssp.1996.0133.

URL https://www.sciencedirect.com/science/article/pii/S0888327096901338

[14] R. Arcangeli, Pseudo-solution de l'équation ax=y, Comptes rendus hebdomadaires des séances de l'académie des sciences serie A 263 (8) (1966) 282-285.

[15] P. V. Overschee, B. L. D. Moor, Subspace Identification for Linear Systems : Theory - Implementation - Applications, Springer, Boston, MA, 1996. doi:https://doi.org/10.1007/ 978-1-4613-0465-4. 
[16] P. Guillaume, P. Verboven, B. Cauberghe, S. Vanlanduit, E. Parloo, G. De Sitter, Frequencydomain system identification techniques for experimental and operational modal analysis, IFAC Proceedings Volumes 36 (16) (2003) 1609-1614, 13th IFAC Symposium on System Identification (SYSID 2003), Rotterdam, The Netherlands, 27-29 August, 2003. doi:https: //doi.org/10.1016/S1474-6670(17)34990-X.

URL https://www.sciencedirect.com/science/article/pii/S147466701734990X

[17] R. J. Allemang, D. L. Brown, A. W. Phillips, R. Allemang, Survey of Modal Techniques Applicable to Autonomous/Semi-Autonomous Parameter Identification, in: Proceedings of ISMA2010 including USD2010, 2010, pp. 3331-3372.

[18] M. I. Friswell, J. E. Mottershead, Finite Element Model Updating in Structural Dynamics, Vol. 38 of Solid Mechanics and its Applications, Springer Netherlands, Dordrecht, 1995. doi: 10.1007/978-94-015-8508-8.

URL http://link.springer.com/10.1007/978-94-015-8508-8

[19] J. E. Mottershead, M. Link, M. I. Friswell, The sensitivity method in finite element model updating: A tutorial, Mechanical Systems and Signal Processing 25 (7) (2011) 2275-2296. doi:10.1016/j.ymssp.2010.10.012.

URL https://linkinghub.elsevier.com/retrieve/pii/S0888327010003316

[20] M. I. Friswell, Damage identification using inverse methods, Philosophical Transactions of the Royal Society A: Mathematical, Physical and Engineering Sciences 365 (1851) (2007) 393-410. doi:10.1098/rsta.2006.1930.

URL https://royalsocietypublishing.org/doi/10.1098/rsta.2006.1930

[21] J. M. Brownjohn, P. Q. Xia, H. Hao, Y. Xia, Civil structure condition assessment by fe model updating: Methodology and case studies, Finite Elements in Analysis and Design 37 (2001) 761-775. doi:10.1016/S0168-874X(00)00071-8.

[22] A. Teughels, G. D. Roeck, Damage detection and parameter identification by finite element model updating, Archives of Computational Methods in Engineering 12 (2005) 123-164. doi: $10.1007 / \mathrm{BF} 03044517$.

[23] B. Weber, P. Paultre, J. Proulx, Structural damage detection using nonlinear parameter identification with Tikhonov regularization, Structural Control and Health Monitoring 14 (3) (2007) 406-427. doi:10.1002/stc.164.

URL https://onlinelibrary.wiley.com/doi/10.1002/stc.164

[24] J. P. Kaipio, E. Somersalo, Statistical inversion theory, Statistical and computational inverse problems (2005) 49-114.

[25] P. Ladevèze, D. Leguillon, Error estimate procedure in the finite element method and applications, SIAM Journal on Numerical Analysis 20 (3) (1983) 485-509. doi:https: //doi.org/10.1137/0720033.

[26] P. Ladevèze, J. P. Pelle, Mastering calculations in linear and nonlinear mechanics, Mechanical engineering series, Springer Science, New York, 2005.

[27] F. Louf, J.-P. Combe, J.-P. Pelle, Constitutive error estimator for the control of contact problems involving friction, Computers \& structures 81 (2003) 1759-1772. doi:10.1016/ s0045-7949(03)00200-1. 
[28] P. Ladevèze, J. Waeytens, Model verification in dynamics through strict upper error bounds, Computer Methods in Applied Mechanics and Engineering 198 (2009) 1775-1784. doi:10. 1016/j.cma.2008.12.020.

[29] P. Ladevèze, L. Chamoin, Éric Florentin, A new non-intrusive technique for the construction of admissible stress fields in model verification, Computer Methods in Applied Mechanics and Engineering 199 (2010) 766-777. doi:10.1016/j.cma.2009.11.007.

[30] F. Pled, L. Chamoin, P. Ladevèze, On the techniques for constructing admissible stress fields in model verification: Performances on engineering examples, International Journal for Numerical Methods in Engineering 88 (2011) 409-441. doi:10.1002/nme.3180.

[31] L. Chamoin, P. Diez, Verification and validation for and with reduced order modeling (2015). URL https://hal.archives-ouvertes.fr/hal-01241728

[32] P. Ladevèze, L. Chamoin, The constitutive relation error method: A general verification tool, in: Verifying Calculations-Forty Years On, Springer, 2016, pp. 59-94. doi:10.1007/ 978-3-319-20553-3_4.

URL https://doi.org/10.1007/978-3-319-20553-3_4

[33] P. Ladevèze, M. Reynier, N. M. M. Maia, Error localization for updating FE models using frequency response functions, in: Proceedings of SPIE - The International Society for Optical Engineering, 1994, pp. 1299-1308.

[34] P. Ladevèze, A. Chouaki, Application of a posteriori error estimation for structural model updating, Inverse Problems 15 (1999) 49-58. doi:10.1088/0266-5611/15/1/009.

[35] A. Deraemaeker, P. Ladevèze, P. Leconte, Reduced bases for model updating in structural dynamics based on constitutive relation error, Computer Methods in Applied Mechanics and Engineering 191 (2002) 2427-2444. doi:10.1016/S0045-7825(01)00421-2.

[36] A. Deraemaeker, P. Ladevèze, T. Romeuf, Model validation in the presence of uncertain experimental data, Engineering Computations (Swansea, Wales) 21 (2004) 808-833. doi: $10.1108 / 02644400410554335$.

[37] P. E. Charbonnel, P. Ladevèze, F. Louf, C. L. Noac'h, A robust CRE-based approach for model updating using in situ measurements, Computers and Structures 129 (2013) 63-73. doi:10.1016/j.compstruc.2013.08.002.

URL http://dx.doi.org/10.1016/j.compstruc.2013.08.002

[38] P. Feissel, O. Allix, Modified constitutive relation error identification strategy for transient dynamics with corrupted data: The elastic case, Computer Methods in Applied Mechanics and Engineering 196 (2007) 1968-1983. doi:10.1016/j.cma.2006.10.005.

[39] K. Hadj-Sassi, Une stratégie d'identification conjointe des parametres et de l'état de structuresa comportements non-linéaires. assimilation de données et erreur en loi de comportement, Ph.D. thesis, Ecole Polytechnique (2007).

[40] W. Aquino, M. Bonnet, Analysis of the error in constitutive equation approach for timeharmonic elasticity imaging, SIAM Journal on Applied Mathematics 79 (3) (2019) 822-849. doi:https://doi.org/10.1137/18M1231237. 
[41] B. Faverjon, J. J. Sinou, Identification of an open crack in a beam using an a posteriori error estimator of the frequency response functions with noisy measurements, European Journal of Mechanics, A/Solids 28 (2009) 75-85. doi:10.1016/j.euromechsol.2008.02.006.

URL http://dx.doi.org/10.1016/j.euromechsol.2008.02.006

[42] E. Barbarella, Towards the localization and characterization of defects based on the modified error in constitutive relation : focus on the buckling test and comparison with other type of experiments., Ph.D. thesis, ENS Cachan (2017).

[43] H. D. Bui, A. Constantinescu, Spatial localization of the error of constitutive law for the identification of defects in elastic bodies, Archives of Mechanics 52 (4-5) (2000) 511-522.

[44] T. A. Silva, N. M. Maia, Detection and localisation of structural damage based on the error in the constitutive relations in dynamics, Applied Mathematical Modelling 46 (2017) 736-749. doi:10.1016/j.apm.2016.07.002.

URL http://dx.doi.org/10.1016/j.apm.2016.07.002

[45] O. Allix, P. Feissel, H. M. Nguyen, Identification strategy in the presence of corrupted measurements, Engineering computations (2005). doi:https://doi.org/10.1108/ 02644400510602989.

[46] H. M. Nguyen, O. Allix, P. Feissel, A robust identification strategy for rate-dependent models in dynamics, Inverse Problems 24 (2008). doi:10.1088/0266-5611/24/6/065006.

[47] M. B. Azzouna, P. Feissel, P. Villon, Robust identification of elastic properties using the modified constitutive relation error, Computer Methods in Applied Mechanics and Engineering 295 (2015) 196-218. doi:10.1016/j.cma.2015.04.004.

[48] M. I. Diaz, W. Aquino, M. Bonnet, A modified error in constitutive equation approach for frequency-domain viscoelasticity imaging using interior data, Computer methods in applied mechanics and engineering 296 (2015) 129-149. doi:https://doi.org/10.1016/j. cma.2015.07.025.

[49] M. Bonnet, W. Aquino, Three-dimensional transient elastodynamic inversion using an error in constitutive relation functional, Inverse Problems 31 (3) (2015) 035010. doi:10.1088/ 0266-5611/31/3/035010.

[50] B. Banerjee, T. F. Walsh, W. Aquino, M. Bonnet, Large scale parameter estimation problems in frequency-domain elastodynamics using an error in constitutive equation functional, Computer Methods in Applied Mechanics and Engineering 253 (2013) 60-72. doi:10.1016/ j.cma.2012.08.023.

URL http://dx.doi.org/10.1016/j.cma.2012.08.023

[51] J. E. Warner, M. I. Diaz, W. Aquino, M. Bonnet, Inverse material identification in coupled acoustic-structure interaction using a modified error in constitutive equation functional, Computational Mechanics 54 (2014) 645-659. doi:10.1007/s00466-014-1018-0.

[52] S. Guchhait, B. Banerjee, Constitutive error based parameter estimation technique for plate structures using free vibration signatures, Journal of Sound and Vibration 419 (2018) 302-317. doi:https://doi.org/10.1016/j.jsv.2018.01.020. 
[53] V. Decouvreur, A. Deraemaeker, P. Ladevèze, P. Bouillard, Building a suited reduced modal basis for updating 3D acoustic models with the constitutive law error method, Computer Methods in Applied Mechanics and Engineering 196 (2007) 3400-3408. doi:10.1016/j.cma. 2007.03 .006$.

[54] V. Decouvreur, P. Ladevèze, P. Bouillard, Updating 3D acoustic models with the constitutive relation error method: A two-stage approach for absorbing material characterization, Journal of Sound and Vibration 310 (2008) 985-997. doi:10.1016/j.jsv.2007.08.012.

[55] H. Oliveira, F. Louf, E. Hervé-Secourgeon, F. Gatuingt, Wall-slab joint parameter identification of a reinforced concrete structure using possibly corrupted modal data, International Journal for Numerical and Analytical Methods in Geomechanics 44 (2020) 19-39. doi:10.1002/nag.2994.

[56] B. Marchand, L. Chamoin, C. Rey, Real-time updating of structural mechanics models using kalman filtering, modified constitutive relation error, and proper generalized decomposition, International Journal for Numerical Methods in Engineering (2015) 1102-1119doi:10.1002/ nme.5197.

URL http://onlinelibrary.wiley.com/doi/10.1002/nme.3279/full

[57] R. Bouclier, F. Louf, L. Chamoin, Real-time validation of mechanical models coupling PGD and constitutive relation error, Computational Mechanics 52 (2013) 861-883. doi:10.1007/ s00466-013-0850-y.

[58] B. Marchand, L. Chamoin, C. Rey, Parameter identification and model updating in the context of nonlinear mechanical behaviors using a unified formulation of the modified constitutive relation error concept, Computer Methods in Applied Mechanics and Engineering 345 (2019) 1094-1113. doi:10.1016/j.cma.2018.09.008.

URL https://doi.org/10.1016/j.cma.2018.09.008

[59] P. Stoica, R. L. Moses, Spectral analysis of signals (2005).

[60] P.-E. Charbonnel, Space launcher model-updating from in-situ measurements (in french)., Ph.D. thesis, ENS Cachan (2012).

[61] R. J. Guyan, Reduction of stiffness and mass matrices, AIAA journal 3 (2) (1965) 380-380.

[62] G. Puel, Sur une théorie des méconnaissances en dynamique des structures, Ph.D. thesis, ENS Cachan (2005).

[63] C. Shih, Y. Tsuei, R. Allemang, D. Brown, Complex mode indication function and its applications to spatial domain parameter estimation, Mechanical systems and signal processing 2 (4) (1988) 367-377. doi:https://doi .org/10.1016/0888-3270 (88)90060-X.

[64] C. G. Broyden, The Convergence of a Class of Double-rank Minimization Algorithms: 2. The New Algorithm, IMA Journal of Applied Mathematics 6 (3) (1970) 222-231. arXiv: https://academic.oup.com/imamat/article-pdf/6/3/222/1848059/6-3-222.pdf, doi: 10.1093/imamat/6.3.222.

URL https://doi.org/10.1093/imamat/6.3.222 
[65] R. Fletcher, A new approach to variable metric algorithms, The Computer Journal 13 (3) (1970) 317-322. arXiv: https ://academic.oup.com/comjnl/article-pdf/13/3/317/ 988678/130317.pdf, doi:10.1093/comjnl/13.3.317.

URL https://doi.org/10.1093/comjnl/13.3.317

[66] D. Goldfarb, A family of variable-metric methods derived by variational means, Mathematics of computation 24 (109) (1970) 23-26. doi:https://doi.org/10.1090/ S0025-5718-1970-0258249-6.

[67] D. F. Shanno, Conditioning of quasi-newton methods for function minimization, Mathematics of computation 24 (111) (1970) 647-656. doi:https://doi.org/10.1090/ S0025-5718-1970-0274029-X.

[68] Cast3M, http://www-cast3m.cea.fr (2020).

[69] SEISM Institute, https://www.institut-seism.fr (2012).

[70] A. Alarcon, Une approche de l'identification en dynamique des structures combinant l'erreur en relation de comportement et le filtrage de kalman, Ph.D. thesis, Ecole Polytechnique (2012).

[71] W. Bangerth, A. Joshi, Adaptive finite element methods for the solution of inverse problems in optical tomography, Inverse Problems 24 (6 2008). doi:10.1088/0266-5611/24/3/034011.

[72] W. Bangerth, A framework for the adaptive finite element solution of large-scale inverse problems, SIAM Journal on Scientific Computing 30 (2008) 2965-2989. doi:10.1137/070690560.

[73] G. Puel, D. Aubry, Using mesh adaption for the identification of a spatial field of material properties, International Journal for Numerical Methods in Engineering 88 (2011) 205-227. doi:10.1002/nme.3170.

[74] R. Becker, B. Vexler, Mesh refinement and numerical sensitivity analysis for parameter calibration of partial differential equations, Journal of Computational Physics 206 (2005) 95-110. doi:10.1016/j.jcp.2004.12.018.

[75] L. Chamoin, P. Diéz, Verifying simulations, Springer, 2016. doi:10.1007/ 978-3-319-20553-3. 